Provided for non-commercial research and education use. Not for reproduction, distribution or commercial use.

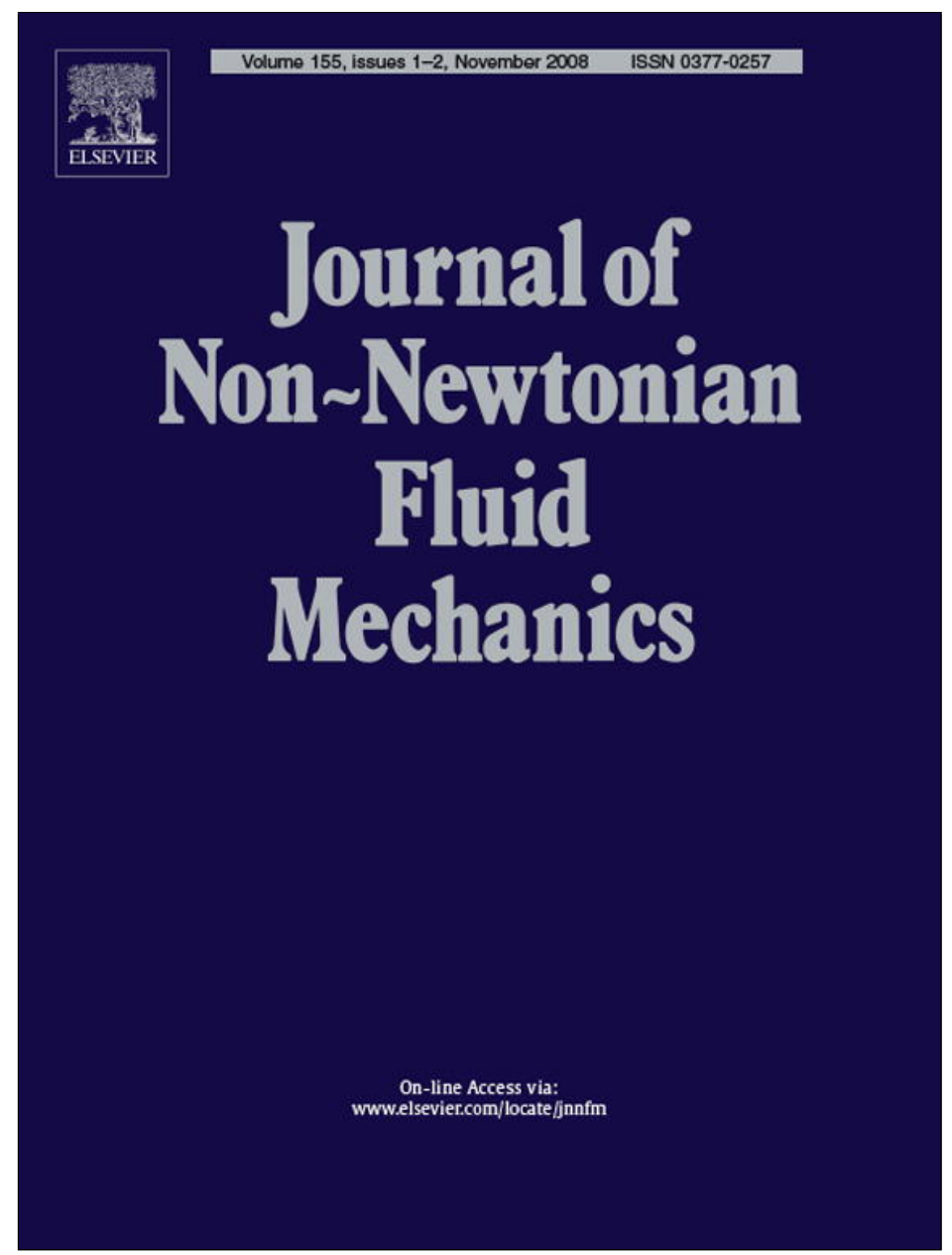

This article appeared in a journal published by Elsevier. The attached copy is furnished to the author for internal non-commercial research and education use, including for instruction at the authors institution and sharing with colleagues.

Other uses, including reproduction and distribution, or selling or licensing copies, or posting to personal, institutional or third party websites are prohibited.

In most cases authors are permitted to post their version of the article (e.g. in Word or Tex form) to their personal website or institutional repository. Authors requiring further information regarding Elsevier's archiving and manuscript policies are encouraged to visit:

http://www.elsevier.com/copyright 


\title{
A parallel adaptive unstructured finite volume method for linear stability (normal mode) analysis of viscoelastic fluid flows
}

\author{
Mehmet Sahin*, Helen J. Wilson \\ Department of Mathematics, UCL, Gower Street, London WC1E 6BT, UK \\ Received 29 November 2007; accepted 18 January 2008
}

\begin{abstract}
A parallel unstructured finite volume method is presented for analysis of the stability of two-dimensional steady Oldroyd-B fluid flows to small amplitude three-dimensional perturbations. A semi-staggered dilation-free finite volume discretization with Newton's method is used to compute steady base flows. The linear stability problem is treated as a generalized eigenvalue problem (GEVP) in which the rightmost eigenvalue determines the stability of the base flow. The rightmost eigenvalues associated with the most dangerous eigenfunctions are computed through the use of the shift-invert Arnoldi method. To avoid fine meshing in the regions where the flow variables are changing slowly, a local mesh refinement technique is used in order to increase numerical accuracy with a lower computational cost. The CUBIT mesh generation environment has been used to refine the quadrilateral meshes locally. In order to achieve higher performance on parallel machines the algebraic systems of equations resulting from the steady problem and the GEVP have been solved by implementing the MUltifrontal Massively Parallel Solver (MUMPS). The proposed method is applied to the linear stability analysis of the flow of an Oldroyd-B fluid past a linear periodic array of circular cylinders in a channel and a linear array of circular half cylinders placed on channel walls. Two different leading eigenfunctions are identified for close and wide cylinder spacing for the periodic array of cylinders. The numerical results indicate good agreement with the numerical and experimental results available in the literature.
\end{abstract}

Crown Copyright (0) 2008 Published by Elsevier B.V. All rights reserved.

Keywords: Viscoelastic fluid flow; Normal mode analysis; Finite volume method; Unstructured methods; Mesh adaptation

\section{Introduction}

Accurate prediction of instabilities in viscoelastic flows presents one of the most challenging problems in nonNewtonian fluid mechanics, and has a crucial importance for polymer processing, where output quality constraints require that operating conditions should be maintained in the stable flow regime. In the past, instabilities in Taylor-Couette flows, instabilities in cone-and-plate and plate-and-plate flows and instabilities in parallel shear flows with interfaces have been extensively studied. Extensive reviews of viscoelastic instabilities are given by Larson [20] and Shaqfeh [33]. These viscoelastic instabilities occur in the creeping motion of nonNewtonian polymeric liquids and are entirely absent in the

\footnotetext{
* Corresponding author. Current address: Department of Aerospace Engineering Sciences, University of Colorado, Boulder, CO 80309, USA.

E-mail address: msahin.ae00@gtalumni.org (M. Sahin).
}

corresponding motion of Newtonian liquids. McKinley et al. [23] and Pakdel and McKinley [28] suggested that for shear-dominated flows one destabilizing mechanism was a combination of streamline curvature and large elastic stresses along the streamlines, giving rise to an extra hoop stress in a direction normal to the streamlines, which can lead to instabilities.

In this work we present an efficient numerical scheme based on the semi-staggered finite volume method [30,32] for the linear stability analysis of two-dimensional steady viscoelastic fluid flows to small amplitude, three-dimensional perturbations. The steady-state base flow solutions are obtained using a Newton's method. The linear stability problem is treated as a generalized eigenvalue problem (GEVP) in which the rightmost eigenvalue determines the stability of the base flow. To compute a subset of the eigenvalues corresponding to the rightmost eigenvalues (associated with the most dangerous eigenfunctions) we use the shift-invert Arnoldi method [4,29]. The implementation of LU algorithm in the steady problem and the GEVP of this paper was carried out using the MUltifrontal Massively Parallel Solver 
(MUMPS) of Amestoy et al. [1,2]. The multifrontal method used is a direct method based on LU decomposition for the solution of general sparse systems of linear equations with several different matrix reordering algorithms such as approximate minimum degree, minimum fill-in, etc. The principal feature of a multifrontal method is that the overall factorization is driven by an assembly tree. The approach employed by MUMPS combines partial static scheduling of the task dependency graph during the symbolic factorization and distributed dynamic scheduling during the numerical factorization to equilibrate work among the processors. In our work, the use of LU decomposition (for the given problem sizes of this paper) is particularly efficient for the shift-invert Arnoldi method where we solve the same system of linear equations for each Krylov subspace dimension. Communication between processors uses the message passing interface (MPI) standard.

Accurate predictions of complex viscoelastic fluid flows are highly demanding because they are required to resolve physical features such as geometric singularities, viscoelastic boundary layers and wakes. These features are generally associated with steep gradients in the flow variables, embedded in or adjacent to regions where these flow variables change more smoothly. An adaptive mesh technique takes advantage of these low gradient regions in order to avoid a fine meshing of the entire computational domain. Therefore, mesh adaptation is a useful technique for increasing accuracy at a lower computational cost. Considerable effort has been given to the implementation of adaptive procedures and a posteriori error estimates for finite element and finite volume methods. Recently, several a posteriori error estimates for viscoelastic fluid flows have been given [25,11,14]. These algorithms are combined with a remeshing algorithm based on point enrichment through successive local subdivision of elements or changing the order of the interpolating function. However, point enrichment through successive local subdivision of quadrilateral elements results in an irregular change in element sizes compared to neighbouring elements throughout the domain, and the refined elements are not necessarily aligned with steep flow gradients such as viscoelastic wakes and boundary layers. Therefore, we have chosen to refine our mesh with a user defined input. The CUBIT [7,27] mesh generation environment has been used to split the quadrilateral elements. The use of local subdivision of quadrilateral elements on anisotropic meshes will allow us to reduce mesh sizes by several orders of magnitude. An isotropic remeshing technique which we had developed based on the gradient of the principal stress difference was not able to reach the same order of mesh size, because of highly elongated viscoelastic wakes. The efficiency of the isotropic remeshing technique would need to be improved further allowing the use of anisotropic meshes similar to that of Fortin et al. [15].

In this study, we apply our numerical scheme to examine the linear stability of the flow of an Oldroyd-B fluid through a linear array of cylinders confined in channel. Viscoelastic flow past a periodic array of cylinders has received considerable attention. Talwar and Khomami [39] used numerical simulations to study steady creeping and inertial flow of a shear thinning viscoelastic fluid past a periodic square array of cylinders using a high-order Galerkin finite volume method with a variety of nonlinear dif- ferential constitutive equations. Souvaliotis and Beris [37] used a domain decomposition spectral collocation method to simulate viscoelastic flows through a square array of cylinders and a single row of cylinders. Liu et al. [22] presented numerical simulations for the flow of polymer solutions around a periodic linear array of cylinders with both wide and close spacing between the cylinders using three different constitutive equations derived from kinetic theory of dilute polymer solutions: the Giesekus, FENE-P and FENE-CR models. Georgiou et al. [16] used both numerical and experimental simulations to study Newtonian and non-Newtonian flow obstructed by the antisymmetric positioning of an array of equally spaced cylinders in a channel. Chmielewski and Jayaraman [12] experimentally studied the kinematics of viscous and viscoelastic fluid flows through square and hexagonal arrays of circular cylinders with a porosity of $70 \%$. Khomami and Moreno [19] studied low Reynolds number flow of Newtonian and viscoelastic Boger fluids past a periodic square array of cylinders with a porosity of 0.45 and 0.86 . Liu [21] also experimentally studied the flow of polymer solutions around a periodic linear array of cylinders with both wide and close spacing between the cylinders. Experimental studies for a single cylinder in a channel have been reported by McKinley et al. [24] and Shiang et al. [34]. For a single cylinder in a channel McKinley et al. [24] and Byars [9] have discovered and studied a transition to a steady three-dimensional cellular pattern that develops in the wake of the cylinder at a critical Weissenberg number. The onset of flow instability is accompanied by enhanced flow resistance. Liu [21] found a transition from a two-dimensional flow to a three-dimensional, time-dependent asymmetric flow for closely spaced periodic array of cylinders in a channel at a critical Weissenberg number. Chmielewski and Jayaraman [12] and Khomami and Moreno [19] also observed similar instabilities for square arrays of circular cylinders by using flow visualization.

Early numerical investigations of the linear stability properties of the flow of an Oldroyd-B fluid through a linear array of cylinders in a channel have resulted with only limited success $[38,35]$. In a later study, Smith et al. [36] investigated the linear stability of a two-dimensional steady base flow to threedimensional infinitesimal perturbations both by time integration of the linearized evolution equations for infinitesimal perturbations and by an eigenvalue analysis based on the Arnoldi method. The flow of an Oldroyd-B fluid through a closely spaced periodic array of cylinders $(L=2.5 R)$ was found to be unstable to threedimensional perturbations at a critical Weissenberg number that is in remarkably good agreement with the experimental observations by Liu [21]. The wavenumber of the disturbance in the normal direction is also in good agreement with the experimental measurements. The only discrepancy between experimental and numerical works is the leading eigenfunction which is found in the numerical study to be a steady symmetric state while the experimental results indicate a periodic asymmetric state. However, the authors point out that the leading eigenfunction is extremely sensitive to geometrical imperfections, which may lead to asymmetric flow structures similar to those observed experimentally. The authors in [36] have also carried out additional calculations in order to determine the scaling of 
the critical Weissenberg number with the cylinder spacing $L$. The calculations up to $L=4.0 R$ indicate a increase in critical Weissenberg number with the cylinder spacing. However, the experimental results of Liu [21] indicate an opposite trend.

In the current paper we have combined our unstructured finite volume code with local mesh refinement in order to investigate the linear stability of two-dimensional viscoelastic fluid flows to small amplitude, three-dimensional perturbations. Our calculations for closely spaced cylinders indicate a symmetric leading eigenfunction similar to the work of Smith et al. [36]. However, the second eigenvalue, which has an asymmetric eigenfunction, is so close to the leading eigenvalue that the two eigenvalues cross into instability almost simultaneously with any increase in Weissenberg number, leading to an asymmetric flow state. In addition, the calculations for widely spaced circular cylinders have indicated a leading eigenfunction which differs completely from that of the closely spaced cylinders. The leading eigenfunctions for widely spaced circular cylinders indicate large spanwise velocity components confined to the very small region behind the cylinders. This large spanwise velocity component leads to the merging of streamtraces behind the circular cylinders, which is similar to the experimental observations of McKinley et al. [24] and Shiang et al. [34]. Even though the calculations for close cylinder spacing show an increase in the critical Weissenberg number with increasing cylinder spacing, the calculations at higher cylinder spacing indicate an opposite trend as in the experimental work of Liu [21]. Unfortunately, we could not exactly compute the critical Weissenberg numbers due to the classical high Weissenberg number problem for large cylinder spacing. In the literature, the only experimental results available are those of Liu [21], and the author investigated only two cylinder spacings: $L=2.5 R$ and $6.0 R$. Therefore, it is not possible to draw any conclusion using these two points as in Fig. 13 of [36]. Normally we would expect two different stability curves corresponding to close and wide cylinder spacing. Further experimental and numerical work is needed in order to clarify the issue. In addition to the confined periodic linear array of circular cylinders, we have presented numerical results for a linear array of circular half cylinders placed on channel walls, which is relevant to contraction-expansion flows. The calculations have indicated an increase in the critical Weissenberg number and a decrease in wavenumber in the third dimension with increasing spacing between the cylinders.

The paper is organized as follows: In Section 2 the governing equations and the numerical method are briefly given. In Section
3 the proposed method is applied to the linear stability analysis of the flow of an Oldroyd-B fluid through a linear array of cylinders in a channel and a linear array of circular half cylinders placed on the channel walls. The leading eigenfunctions are identified for both wide and close spacing between the cylinders. Conclusions are presented in Section 4.

\section{Mathematical and numerical formulation}

The governing equations for three-dimensional unsteady flow of an incompressible Oldroyd-B fluid can be written in dimensionless form as follows: the continuity equation

$-\nabla \cdot \mathbf{u}=0$

the momentum equations:

$\operatorname{Re}\left[\frac{\partial \mathbf{u}}{\partial t}+(\mathbf{u} \cdot \nabla) \mathbf{u}\right]+\nabla p=\beta \nabla^{2} \mathbf{u}+\nabla \cdot \mathbf{T}$

and the constitutive equation for the Oldroyd-B model:

$$
\begin{aligned}
& W e\left[\frac{\partial \mathbf{T}}{\partial t}+(\mathbf{u} \cdot \nabla) \mathbf{T}-(\nabla \mathbf{u})^{\top} \cdot \mathbf{T}-\mathbf{T} \cdot \nabla \mathbf{u}\right] \\
& \quad=(1-\beta)\left(\nabla \mathbf{u}+\nabla \mathbf{u}^{\top}\right)-\mathbf{T}
\end{aligned}
$$

In these equations $\mathbf{u}$ represents the velocity vector, $p$ is the pressure and $\mathbf{T}$ is the extra stress tensor. The dimensionless parameters are the Reynolds number $R e$, the Weissenberg number $W e$ and the viscosity ratio $\beta$. The steady state twodimensional base flow and the linear stability equations are computed by using the dilation-free semi-staggered finite volume method given in detail in [30,32].

\subsection{Newton's method}

The steady form of the above equations is solved using Newton's method: discretizing in time using the following form:

$q^{n+1}=q^{n}+\delta q^{n+1}$

for $q$ representing each of the variables $\mathbf{u}, \mathbf{T}$ and $p$, and integrating the differential equations (1) and (3) over a quadrilateral element $\Omega_{e}$ with boundary $\partial \Omega_{e}$ gives

$$
\oint_{\partial \Omega_{e}} \mathbf{n} \cdot \delta \mathbf{u}^{n+1} \mathrm{~d} S=-\oint_{\partial \Omega_{e}} \mathbf{n} \cdot \mathbf{u}^{n} \mathrm{~d} S
$$

$$
\begin{aligned}
& W e\left[\oint_{\partial \Omega_{e}}\left(\mathbf{n} \cdot \delta \mathbf{u}^{n+1}\right) \mathbf{T}^{n} \mathrm{~d} S\right]-W e\left[\int_{\Omega_{e}}\left[\left(\nabla \delta \mathbf{u}^{n+1}\right)^{\top} \cdot \mathbf{T}^{n}+\mathbf{T}^{n} \cdot \nabla \delta \mathbf{u}^{n+1}\right] \mathrm{d} V\right]+W e\left[\oint_{\partial \Omega_{e}}\left(\mathbf{n} \cdot \mathbf{u}^{n}\right) \delta \mathbf{T}^{n+1} \mathrm{~d} S\right] \\
& -W e\left[\int_{\Omega_{e}}\left[\left(\nabla \mathbf{u}^{n}\right)^{\top} \cdot \delta \mathbf{T}^{n+1}+\delta \mathbf{T}^{n+1} \cdot \nabla \mathbf{u}^{n}\right] \mathrm{d} V\right]-(1-\beta) \oint_{\partial \Omega_{e}}\left[\delta \mathbf{u}^{n+1} \mathbf{n}+\mathbf{n} \delta \mathbf{u}^{n+1}\right] \mathrm{d} S+\int_{\Omega_{e}} \delta \mathbf{T}^{n+1} \mathrm{~d} V \\
& \quad=-W e\left[\oint_{\partial \Omega_{e}}\left(\mathbf{n} \cdot \mathbf{u}^{n}\right) \mathbf{T}^{n} \mathrm{~d} S\right]+W e\left[\int_{\Omega_{e}}\left[\left(\nabla \mathbf{u}^{n}\right)^{\top} \cdot \mathbf{T}^{n}+\mathbf{T}^{n} \cdot \nabla \mathbf{u}^{n}\right] \mathrm{d} V\right]+(1-\beta) \oint_{\partial \Omega_{e}}\left[\mathbf{u}^{n} \mathbf{n}+\mathbf{n} \mathbf{u}^{n}\right] \mathrm{d} S-\int_{\Omega_{e}} \mathbf{T}^{n} \mathrm{~d} V
\end{aligned}
$$


and integrating the differential equation (2) over an arbitrary irregular dual control volume $\Omega_{d}$ with boundary $\partial \Omega_{d}$ gives

$$
\begin{aligned}
& \operatorname{Re}\left[\oint_{\partial \Omega_{d}}\left(\mathbf{n} \cdot \delta \mathbf{u}^{n+1}\right) \mathbf{u}^{n} \mathrm{~d} S+\oint_{\partial \Omega_{d}}\left(\mathbf{n} \cdot \mathbf{u}^{n}\right) \delta \mathbf{u}^{n+1} \mathrm{~d} S\right] \\
& +\oint_{\partial \Omega_{d}} \delta p^{n+1} \mathbf{n} \mathrm{d} S-\beta \oint_{\partial \Omega_{d}} \mathbf{n} \cdot \nabla \delta \mathbf{u}^{n+1} \mathrm{~d} S-\oint_{\partial \Omega_{d}} \mathbf{n} \cdot \delta \mathbf{T}^{n+1} \mathrm{~d} S \\
& =-\oint_{\partial \Omega_{d}}\left(\mathbf{n} \cdot \mathbf{u}^{n}\right) \mathbf{u}^{n} \mathrm{~d} S-\oint_{\partial \Omega_{d}} p^{n} \mathbf{n} \mathrm{d} S \\
& +\beta \oint_{\partial \Omega_{d}} \mathbf{n} \cdot \nabla \mathbf{u}^{n} \mathrm{~d} S+\oint_{\partial \Omega_{d}} \mathbf{n} \cdot \mathbf{T}^{n} \mathrm{~d} S
\end{aligned}
$$

Here $\mathbf{n}$ represents the outward normal unit vector. Fig. 1 illustrates typical four node quadrilateral elements with a dual finite volume constructed by connecting the centroids $c_{i}$ of the elements which share a common vertex. The discrete contribution from cell $c_{1}$ to cell $c_{2}$ for the left hand side of the momentum equation (7) is given by

$$
\begin{aligned}
& \operatorname{Re}\left[\mathbf{n}_{12} \cdot\left(\frac{\delta \mathbf{u}_{1}^{n}+\delta \mathbf{u}_{2}^{n}}{2}\right)\right]\left(\frac{\mathbf{u}_{1}^{n+1}+\mathbf{u}_{2}^{n+1}}{2}\right) S_{12} \\
& +\operatorname{Re}\left[\mathbf{n}_{12} \cdot\left(\frac{\mathbf{u}_{1}^{n}+\mathbf{u}_{2}^{n}}{2}\right)\right]\left(\frac{\delta \mathbf{u}_{1}^{n+1}+\delta \mathbf{u}_{2}^{n+1}}{2}\right) S_{12} \\
& +\mathbf{n}_{12}\left(\frac{\delta p_{1}^{n+1}+\delta p_{2}^{n+1}}{2}\right) S_{12} \\
& -\beta \mathbf{n}_{12} \cdot\left(\frac{\nabla \delta \mathbf{u}_{1}^{n+1}+\nabla \delta \mathbf{u}_{2}^{n+1}}{2}\right) S_{12} \\
& -\mathbf{n}_{12} \cdot\left(\frac{\delta \mathbf{T}_{1}^{n+1}+\delta \mathbf{T}_{2}^{n+1}}{2}\right) S_{12}
\end{aligned}
$$

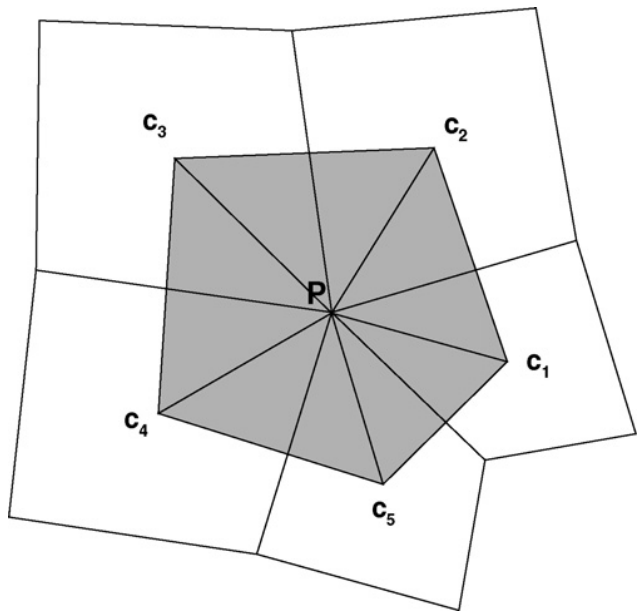

Fig. 1. Two-dimensional unstructured mesh with a dual control volume surrounding a node $P$. where $S_{12}$ is the length between the points $c_{1}$ and $c_{2}$. The other contributions are calculated in a similar manner. The velocity vector at the element centroids $c_{i}$ is computed from the element vertex values using simple averages and the gradient of velocity components $\nabla \delta \mathbf{u}$ are calculated from Green's Theorem:

$\nabla \delta \mathbf{u}_{i}^{n+1}=\frac{1}{V} \oint_{\partial \Omega_{e}} \mathbf{n} \delta \mathbf{u}^{n+1} \mathrm{~d} S$

where the line integral on the right-hand side of Eq. (9) is evaluated using the mid-point rule on each of the element faces. The continuity equation is integrated in a similar manner within each element. The constitutive equation is integrated within each element assuming that the extra stresses $\mathbf{T}_{i}$ and velocity gradients $\nabla \mathbf{u}_{i}$ are constant:

$$
\begin{aligned}
& W e\left[\sum_{m=1}^{4}\left(\mathbf{n} \cdot \delta \mathbf{u}^{n+1}\right) \mathbf{T}_{m}^{n} S\right]-W e\left[\left(\nabla \delta \mathbf{u}_{i}^{n+1}\right)^{\top} \cdot \mathbf{T}_{i}^{n}\right. \\
& \left.+\mathbf{T}_{i}^{n} \cdot \nabla \delta \mathbf{u}_{i}^{n+1}\right] V+W e\left[\sum_{m=1}^{4}\left(\mathbf{n} \cdot \mathbf{u}^{n}\right) \delta \mathbf{T}_{m}^{n+1} S\right] \\
& -W e\left[\left(\nabla \mathbf{u}_{i}^{n}\right)^{\top} \cdot \delta \mathbf{T}_{i}^{n+1}+\delta \mathbf{T}_{i}^{n+1} \cdot \nabla \mathbf{u}_{i}^{n}\right] V \\
& -(1-\beta) \sum_{m=1}^{4}\left[\delta \mathbf{u}^{n+1} \mathbf{n}+\mathbf{n} \delta \mathbf{u}^{n+1}\right] S+\delta \mathbf{T}_{i}^{n+1} V \\
& =-W e\left[\sum_{m=1}^{4}\left(\mathbf{n} \cdot \mathbf{u}^{n}\right) \mathbf{T}_{m}^{n} S\right]+W e\left[\left(\nabla \mathbf{u}_{i}^{n}\right)^{\top} \cdot \mathbf{T}_{i}^{n}\right. \\
& \left.+\mathbf{T}_{i}^{n} \cdot \nabla \mathbf{u}_{i}^{n}\right] V+(1-\beta) \sum_{m=1}^{4}\left[\mathbf{u}^{n} \mathbf{n}+\mathbf{n} \mathbf{u}^{n}\right] S-\mathbf{T}_{i}^{n} V
\end{aligned}
$$

where $\mathbf{T}_{m}$ is the value of the extra stress at the face centres of the quadrilateral elements. In order to extrapolate the extra stresses to the boundaries of the finite volume elements a secondorder upwind least square interpolation $[6,3]$ is used in order to maintain stability. The use of this least square approximation to the gradient term in order to compute the convective term results in the same coefficients computed from the second-order upwind interpolation on uniform Cartesian meshes. Therefore, our approximation is second-order similar to the second-order upwind interpolation.

\subsection{Linear stability (normal mode) analysis}

In this paper, we consider three-dimensional infinitesimal perturbations to the two-dimensional base flow in order to examine the stability of the base flow to disturbances. Each variable is written as a sum of the base state and a small amplitude 
disturbance:

$$
\begin{array}{ll}
T_{x x}=T_{x x}(x, y)+ & \mathrm{i} \hat{\tau}_{x x}(x, y) e^{\mathrm{i} k z+\sigma t} \\
T_{y y}=T_{y y}(x, y)+ & \mathrm{i} \hat{\tau}_{y y}(x, y) e^{\mathrm{i} k z+\sigma t} \\
T_{z z}= & \mathrm{i} \hat{\tau}_{z z}(x, y) e^{\mathrm{i} k z+\sigma t} \\
T_{x y}=T_{x y}(x, y)+ & \mathrm{i} \hat{\tau}_{x y}(x, y) e^{\mathrm{i} k z+\sigma t} \\
T_{x z}= & \hat{\tau}_{x z}(x, y) e^{\mathrm{i} k z+\sigma t} \\
T_{y z}= & \hat{\tau}_{y z}(x, y) e^{\mathrm{i} k z+\sigma t} \\
u=U(x, y)+ & \mathrm{i} \hat{u}(x, y) e^{\mathrm{i} k z+\sigma t} \\
v=V(x, y)+ & \mathrm{i} \hat{v}(x, y) e^{\mathrm{i} k z+\sigma t} \\
w= & \hat{w}(x, y) e^{\mathrm{i} k z+\sigma t} \\
p=P(x, y)+ & \mathrm{i} \hat{p}(x, y) e^{\mathrm{i} k z+\sigma t}
\end{array}
$$

where $\mathrm{i}$ is the imaginary unit, $k$ is the spanwise wave number and $\sigma$ denotes the complex growth rate. The reason we use an imaginary amplitude in some of the normal modes is to ensure a real matrix in the calculation of the generalized eigenvalue eigenproblem, as in the work of Ding and Kawahara [13]. Substituting these equations in the governing equations, subtracting the base flow equations and neglecting quadratic terms we get the following perturbation equations: the continuity equation

$-\nabla \cdot(\hat{u}, \hat{v})-k \hat{w}=0$

the momentum equations:

$$
\begin{aligned}
& \operatorname{Re}[\sigma \hat{u}+(U, V) \cdot \nabla \hat{u}+(\hat{u}, \hat{v}) \cdot \nabla U]+\frac{\partial \hat{p}}{\partial x} \\
& =\beta\left[\nabla^{2} \hat{u}-k^{2} \hat{u}\right]+\frac{1-\beta}{W e}\left[\frac{\partial \hat{\tau}_{x x}}{\partial x}+\frac{\partial \hat{\tau}_{x y}}{\partial y}+k \hat{\tau}_{x z}\right] \\
& \operatorname{Re}[\sigma \hat{v}+(U, V) \cdot \nabla \hat{v}+(\hat{u}, \hat{v}) \cdot \nabla V]+\frac{\partial \hat{p}}{\partial y} \\
& =\beta\left[\nabla^{2} \hat{v}-k^{2} \hat{v}\right]+\frac{1-\beta}{W e}\left[\frac{\partial \hat{\tau}_{x y}}{\partial x}+\frac{\partial \hat{\tau}_{y y}}{\partial y}+k \hat{\tau}_{y z}\right]
\end{aligned}
$$

$$
\begin{aligned}
& \operatorname{Re}[\sigma \hat{w}+(U, V) \cdot \nabla \hat{w}]-k \hat{p} \\
& =\beta\left[\nabla^{2} \hat{w}-k^{2} \hat{w}\right]+\frac{1-\beta}{W e}\left[\frac{\partial \hat{\tau}_{x z}}{\partial x}+\frac{\partial \hat{\tau}_{y z}}{\partial y}-k \hat{\tau}_{z z}\right]
\end{aligned}
$$

the constitutive equation for the Oldroyd-B fluid:

$$
\begin{aligned}
& W e\left[\sigma \hat{\tau}_{x x}+(U, V) \cdot \nabla \hat{\tau}_{x x}+(\hat{u}, \hat{v}) \cdot \nabla T_{x x}\right] \\
& -2 W e\left[\frac{\partial \hat{u}}{\partial x} T_{x x}+\frac{\partial \hat{u}}{\partial y} T_{x y}+\frac{\partial U}{\partial x} \hat{\tau}_{x x}+\frac{\partial U}{\partial y} \hat{\tau}_{x y}\right]=-\hat{\tau}_{x x}
\end{aligned}
$$

$$
\begin{aligned}
& W e\left[\sigma \hat{\tau}_{y y}+(U, V) \cdot \nabla \hat{\tau}_{y y}+(\hat{u}, \hat{v}) \cdot \nabla T_{y y}\right] \\
& -2 W e\left[\frac{\partial \hat{v}}{\partial x} T_{x y}+\frac{\partial \hat{v}}{\partial y} T_{y y}+\frac{\partial V}{\partial x} \hat{\tau}_{x y}+\frac{\partial V}{\partial y} \hat{\tau}_{y y}\right]=-\hat{\tau}_{y y}
\end{aligned}
$$

$W e\left[\sigma \hat{\tau}_{z z}+(U, V) \cdot \nabla \hat{\tau}_{z z}\right]=-\hat{\tau}_{z z}$

$$
\begin{aligned}
& W e\left[\sigma \hat{\tau}_{x y}+(U, V) \cdot \nabla \hat{\tau}_{x y}+(\hat{u}, \hat{v}) \cdot T_{x y}\right] \\
& -W e\left[\frac{\partial \hat{u}}{\partial x} T_{x y}+\frac{\partial \hat{u}}{\partial y} T_{y y}+\frac{\partial U}{\partial x} \hat{\tau}_{x y}+\frac{\partial U}{\partial y} \hat{\tau}_{y y}\right] \\
& -W e\left[\frac{\partial \hat{v}}{\partial x} T_{x x}+\frac{\partial \hat{v}}{\partial y} T_{x y}+\frac{\partial V}{\partial x} \hat{\tau}_{x x}+\frac{\partial V}{\partial y} \hat{\tau}_{x y}\right]=-\hat{\tau}_{x y}
\end{aligned}
$$

$$
\begin{gathered}
W e\left[\sigma \hat{\tau}_{x z}+(U, V) \cdot \nabla \hat{\tau}_{x z}\right]-W e\left[\frac{\partial \hat{w}}{\partial x} T_{x x}+\frac{\partial \hat{w}}{\partial y} T_{x y}\right] \\
-W e\left[\frac{\partial U}{\partial x} \hat{\tau}_{x z}+\frac{\partial U}{\partial y} \hat{\tau}_{y z}\right]=-\hat{\tau}_{x z}
\end{gathered}
$$

$$
\begin{gathered}
W e\left[\sigma \hat{\tau}_{y z}+(U, V) \cdot \nabla \hat{\tau}_{y z}\right]-W e\left[\frac{\partial \hat{w}}{\partial x} T_{x y}+\frac{\partial \hat{w}}{\partial y} T_{y y}\right] \\
-W e\left[\frac{\partial V}{\partial x} \hat{\tau}_{x z}+\frac{\partial V}{\partial y} \hat{\tau}_{y z}\right]=-\hat{\tau}_{y z}
\end{gathered}
$$

where $\nabla$ is the two-dimensional gradient operator because all variables are functions of $x$ and $y$ only. The discretization of Eqs. (12)-(21) follows very closely the ideas employed for the solution of the steady state equations in Section 2.1. However, there are two significant differences between the spatial discretization of the two-dimensional steady base flow equations and that of the three-dimensional perturbation equations. The first is that the three-dimensional perturbation velocity field is not solenoidal $(\nabla \cdot \hat{\mathbf{u}}=-k \hat{w})$ and an additional term appears from the integration of the convective terms $(\hat{\mathbf{u}} \cdot \nabla) \mathbf{U}$ and $(\hat{\mathbf{u}} \cdot \nabla) \mathbf{T}$. The second is that no pressure datum is necessary for three-dimensional perturbations $(k \neq 0)$. The end result of following these procedures is a generalized eigenvalue problem of the form:

$A \mathbf{x}=\sigma B \mathbf{x}$

where the $A$ and $B$ matrices can be formed in a block matrix structure:

$$
\left[\begin{array}{ccc}
A_{\tau \tau} & A_{\tau u} & 0 \\
A_{u \tau} & A_{u u} & A_{u p} \\
0 & A_{p u} & 0
\end{array}\right]\left[\begin{array}{c}
\hat{\tau} \\
\hat{u} \\
\hat{p}
\end{array}\right]=\sigma\left[\begin{array}{ccc}
B_{\tau \tau} & 0 & 0 \\
0 & B_{u u} & 0 \\
0 & 0 & 0
\end{array}\right]\left[\begin{array}{c}
\hat{\tau} \\
\hat{u} \\
\hat{p}
\end{array}\right]
$$

Here $B_{u u}$ becomes zero for Stokes flow and $B_{\tau \tau}$ is a diagonal matrix. The generalized eigenvalue problem (22) is solved by applying the Arnoldi method [4,29] to the equivalent system:

$C \mathbf{x}=\mu \mathbf{x}$

where $C=(A-\lambda B)^{-1} B$ and $\mu=(\sigma-\lambda)^{-1}$. The application of the shift-invert Arnoldi method leads to the construction of an upper Hessenberg matrix $H$ whose eigenvalues (the so-called Ritz values $\hat{\mu}$ ) are approximations to eigenvalues $\mu$ of $C$. From the properties of the Arnoldi method, the best resolution of the spectrum is expected to be around the shift parameter $\lambda$. In the present paper we only use real numbers for the shift parameter, in order to avoid complex arithmetic which increases the computational cost significantly. The Ritz values of $H$ are computed using the Intel Math Kernel Library which uses a multishift form 
of the upper Hessenberg QR algorithm. Solutions to all the discrete algebraic equations that arise in the steady and eigenvalue problems of this paper have been obtained by implementing the MUMPS of Amestoy et al. [1,2].

\section{Numerical results}

In this section the proposed method is applied to the linear stability analysis of the flow of an Oldroyd-B fluid through a linear array of cylinders in a channel and through a linear array of circular half cylinders placed on channel walls. The two-dimensional base flows of the Oldroyd-B fluid are computed using the Newton's method described in Section 2.1. The linear stability of these base flows is analyzed by solving the generalized eigenvalue problem arising from the linear perturbation equations given in Section 2.2. The present numerical calculations have been performed on an SGI Altix 3700 parallel machine with 56 processors (Itanium2 1.3 GHz/3 MB cache processors) available at UCL.

\subsection{Periodic linear array of confined circular cylinders}

Viscoelastic flow past a periodic array of cylinders has received considerable attention and has been studied by many researchers [5,12,16-19,21,22,35-37]. For this flow we consider a circular cylinder of radius $R$ positioned symmetrically between two parallel plates separated by a distance $2 H$. The blockage ratio $R / H$ is set to 0.5 . The computational domain is periodic along the flow direction and the distance between the center of the cylinders is $L$. The dimensionless parameters are the Reynolds number $R e=\langle v\rangle R / \eta$, the Weissenberg number $W e=\lambda\langle v\rangle / R$ and the viscosity ratio $\beta=\eta_{\mathrm{s}} /\left(\eta_{\mathrm{p}}+\eta_{\mathrm{s}}\right)$. The physical parameters are the density $\rho$, the average velocity $\langle v\rangle$, the relaxation time $\lambda$ and the zero-shear-rate viscosity of the fluid $\eta=\eta_{\mathrm{s}}+\eta_{\mathrm{p}}$ where $\eta_{\mathrm{s}}$ is the solvent viscosity. The viscosity ratio $\beta$ is chosen to be 0.67 throughout this paper, which is the value used in the experimental results of Liu [21] for the Oldroyd-B fluid. In this work, periodic boundary conditions are imposed at the inlet boundary and the outlet boundary. No-slip boundary conditions are imposed on all solid walls. The extra stresses are computed everywhere within the computational domain.

The first set of numerical results corresponds to the linear stability of an Oldroyd-B fluid flowing through a linear array of circular cylinders for close cylinder spacing $(L=2.5 R$ and $3.0 R)$. In order to investigate the mesh dependency of the solutions for $L=2.5 R$, four different meshes are employed: mesh M1 with 10,210 node points and 9921 elements, mesh M2 with

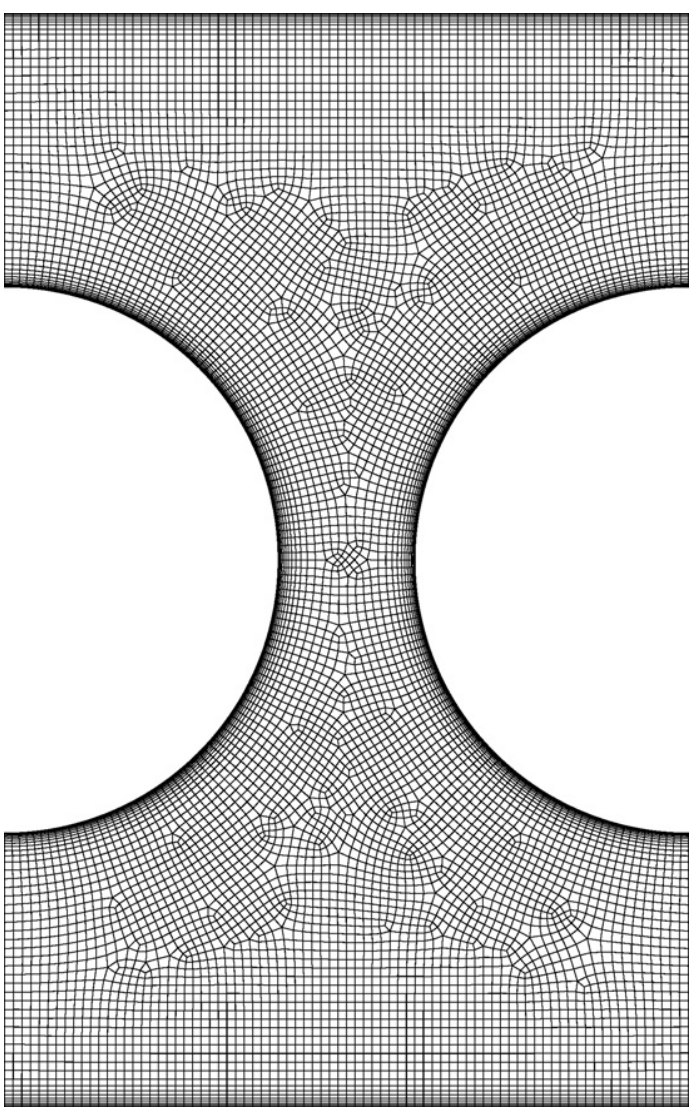

Fig. 2. The computational coarse mesh M1 for the flow past a confined periodic array of circular cylinders for $L=2.5 R$ and $H=4.0 R$ with 10,210 nodes and 9921 elements.

19,027 node points and 18,626 elements, mesh M3 with 36,201 node points and 35,644 elements and mesh M4 with 72,587 node points and 71,800 elements. The successive meshes are generated by multiplying the mesh sizes by $1 / \sqrt{2}$ in each direction and the details of the meshes are given in Table 1. All the meshes are stretched next to the cylinder surface and the lateral solid walls as seen in Fig. 2. To guarantee mesh periodicity during the paving algorithm [8] the mesh scheme at the inlet is copied to the outlet. The steady-state base solutions of the $U$-velocity component, with streamtraces, and the extra stress tensor component $T_{x x}$ are given in Fig. 3 at a Weissenberg number of 1.45 on mesh M4. The calculated streamtraces indicate a pair of counter-rotating separation vortices between the two cylinders. The location of the extreme value of the $U$-velocity component shifts further downstream with an increase in Weissenberg number. The results also indicates large extra stresses

Table 1

Description of quadrilateral meshes used in the present work for $L=2.5 R$ and $H=4.0 R$

\begin{tabular}{lccccc}
\hline Mesh & Number of nodes & Number of elements & $\Delta r_{\min } / R$ & $\Delta S_{\min } / R$ & DOF in Newton \\
\hline M1 & 10,210 & 9,921 & 0.002500 & 0.02618 & 100,104 \\
M2 & 19,027 & 18,626 & 0.001768 & 0.01851 & 112,558 \\
M3 & 36,201 & 35,644 & 0.001250 & 0.01309 & 214,978 \\
M4 & 72,587 & 71,800 & 0.000884 & 0.00925 & 432,374 \\
\hline
\end{tabular}

$\Delta r_{\min }$ and $\Delta S_{\min }$ are the minimum normal and tangential mesh spacing on the cylinder surface. 

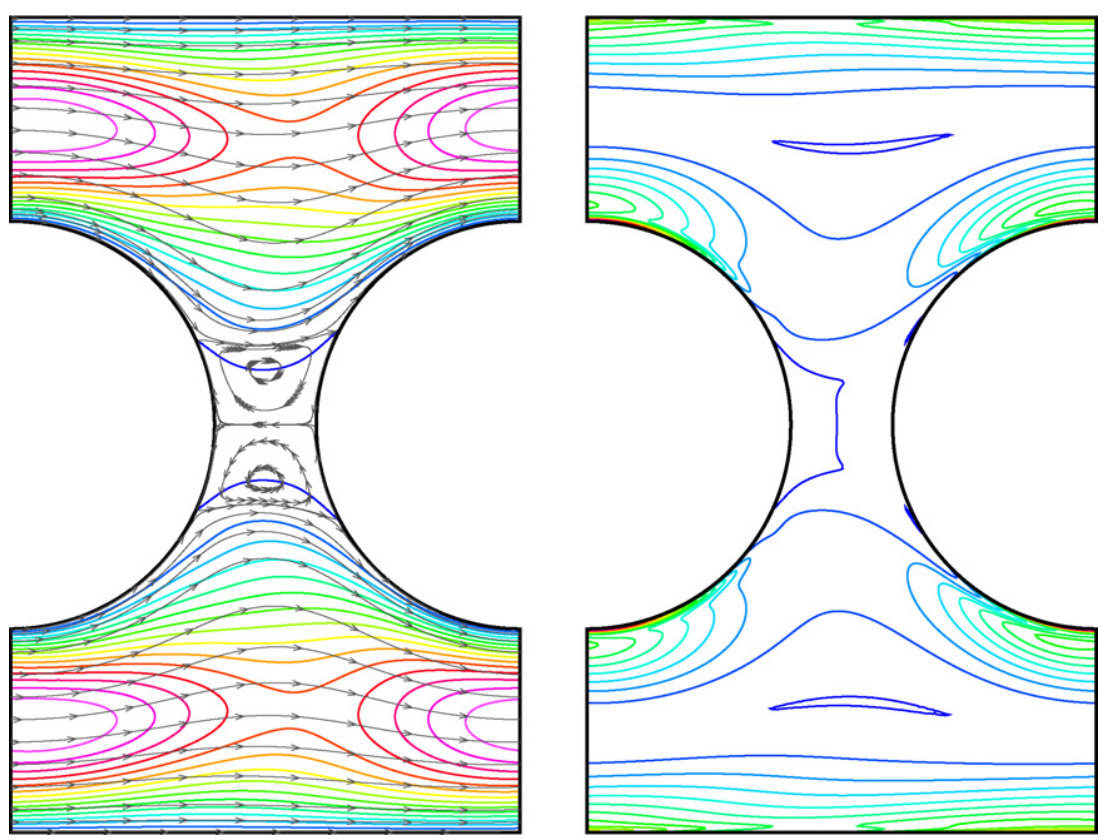

Fig. 3. Computed steady state $U$-velocity contours with streamlines (left) and $T_{x x}$ contour plots (right) on mesh M4 at a subcritical Weissenberg number of 1.45 for an Oldroyd-B fluid $(\beta=0.67)$ with $L=2.5 R$ and $H=4.0 R$. The contour levels shown are from 0 to 3 with an interval of 0.2 for the $U$-velocity and from 0 to 200 with an interval of 10 for $T_{x x}$.

on the solid walls and in the "wake" region across the top of the recirculation region between the cylinders.

For the assessment of solution accuracy, as well as code validation, we supply the values of the first two leading eigenvalues for the Oldroyd-B fluid on meshes M1-M4 with Krylov space dimension $m=80$ and shift parameter $\lambda=1.0$ in Table 2 at a Weissenberg number of 1.45 . The calculations predict two real leading eigenvalues as in the work of Smith et al. [36]. Although the use of a shift parameter value $\lambda=0.0$ would give more accurate results, it may not be possible to capture leading eigenvalues with a large imaginary part if they exist. In addition to the leading eigenvalues, the convergence of the computed eigenspectrum with mesh refinement is shown in Fig. 4 at the same Weissenberg number. We have observed very good mesh convergence in the eigenspectrum around the origin. Using mesh M4, the computed contours of $\hat{\tau}_{x x}$ and the $\hat{w}$-velocity component, with streamtraces, are given for the first two eigenfunctions in Figs. 5 and 6, respectively. The computed first eigenfuction is symmetric about the channel symmetry line, while the second one is antisymmetric, causing a mass flow between the two cylinders as seen in

\section{Table 2}

Convergence of the leading two eigenvalues at a subcritical Weissenberg number of 1.45 with mesh refinement for $L=2.5 R$ and $H=4.0 R$ (Oldroyd-B fluid with $\beta=0.67)$

\begin{tabular}{lllllllll}
\hline Mesh & $m$ & $\lambda$ & $k$ & \multicolumn{2}{c}{ First eigenvalue } & & \multicolumn{2}{c}{ Second eigenvalue } \\
& & & & $\sigma_{R}\left(\times 10^{-2}\right)$ & $\sigma_{I}$ & & $\sigma_{R}\left(\times 10^{-2}\right)$ & $\sigma_{I}$ \\
\hline M1 & 80 & 1.00 & 3.0 & -1.6013 & \pm 0.0000 & -1.8867 & \pm 0.0000 \\
M2 & 80 & 1.00 & 3.0 & -3.0797 & \pm 0.0000 & -3.4278 & \pm 0.0000 \\
M3 & 80 & 1.00 & 3.0 & -3.8116 & \pm 0.0000 & -4.1936 & \pm 0.0000 \\
M4 & 80 & 1.00 & 3.0 & -4.1345 & \pm 0.0000 & -4.5458 & \pm 0.0000 \\
\hline
\end{tabular}

Fig. 6. Although the experimental results of Liu [21] indicated an asymmetric leading eigenfunction, the leading two real eigenvalues are very close to each other and they become positive almost simultaneously with any small increase in Weissenberg number. This eventually leads to an asymmetric base flow, as in the experimental work of Liu [21]. The preferential selection of flow direction between the cylinders is not unique. This may depend on the asymmetry in our meshes. In our calculations we have also used symmetric meshes which also lead to one leading

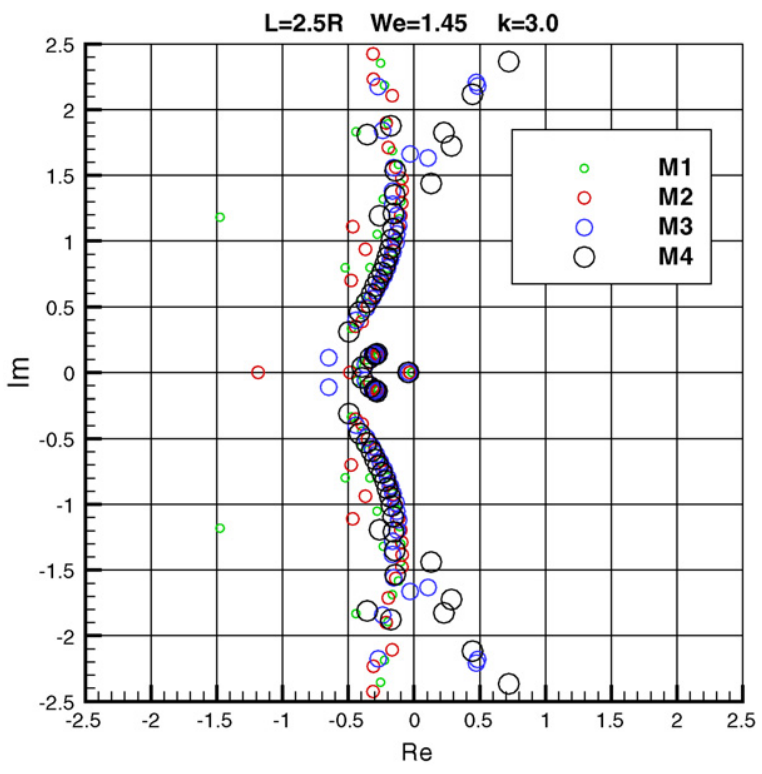

Fig. 4. Convergence of the eigenspectrum at a subcritical Weissenberg number of 1.45 with Krylov space dimension $m=80$ and shift parameter $\lambda=1.0$ for $L=2.5 R$ and $H=4.0 R$ (Oldroyd-B fluid with $\beta=0.67$ ). 

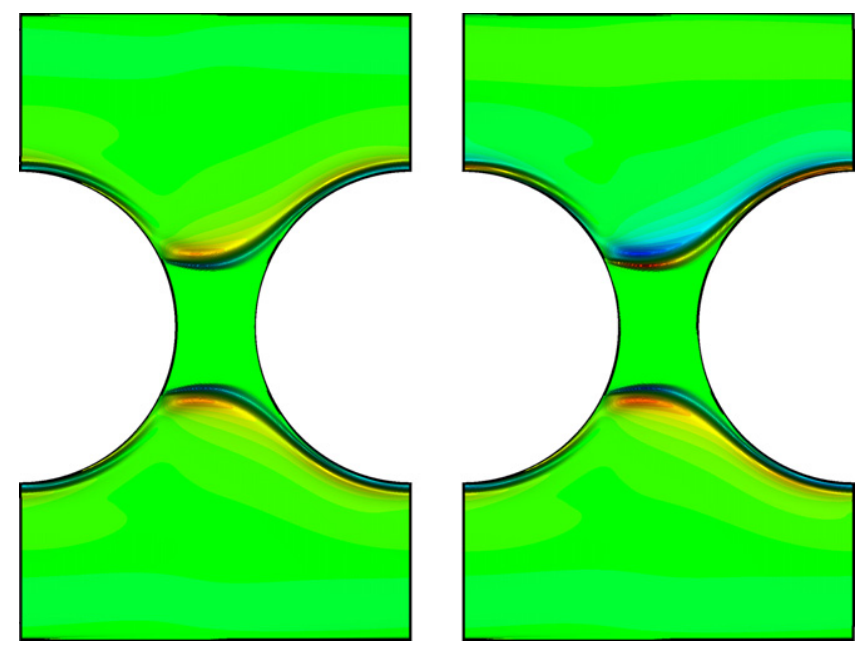

Fig. 5. The computed contours of $\hat{\tau}_{x x}$ for the first two leading eigenfunctions on mesh M4 at a subcritical Weissenberg number of 1.45 with $L=2.5 R$ and $H=4.0 R$. The first eigenfunction (left) is symmetric and the second (right) is antisymmetric (Oldroyd-B fluid with $\beta=0.67$ ).

symmetric eigenfunction and one antisymmetric eigenfunction similar to that seen on the asymmetric meshes. However, we should mention that on the coarse mesh M1 the calculations predict two asymmetric leading eigenfunctions while the symmetric mesh calculations predict one symmetric and one asymmetric leading eigenfunction. In addition, we have increased periodicity to $5.0 R$ for the same problem allowing wavenumbers larger than $L=2.5 R$ in the flow direction. However, this did not alter the numerical results. The convergence of the critical Weissenberg number at which the first leading eigenvalue becomes positive is given in Table 3 for meshes M1-M4. Comparison of the computed critical Weissenberg number and wavenumber with several other results in the literature is provided in Table 4. We observe very good agreement with the experimental results of Liu [21] for both the critical Weissenberg number and the wavenumber $k$ in the third dimension.
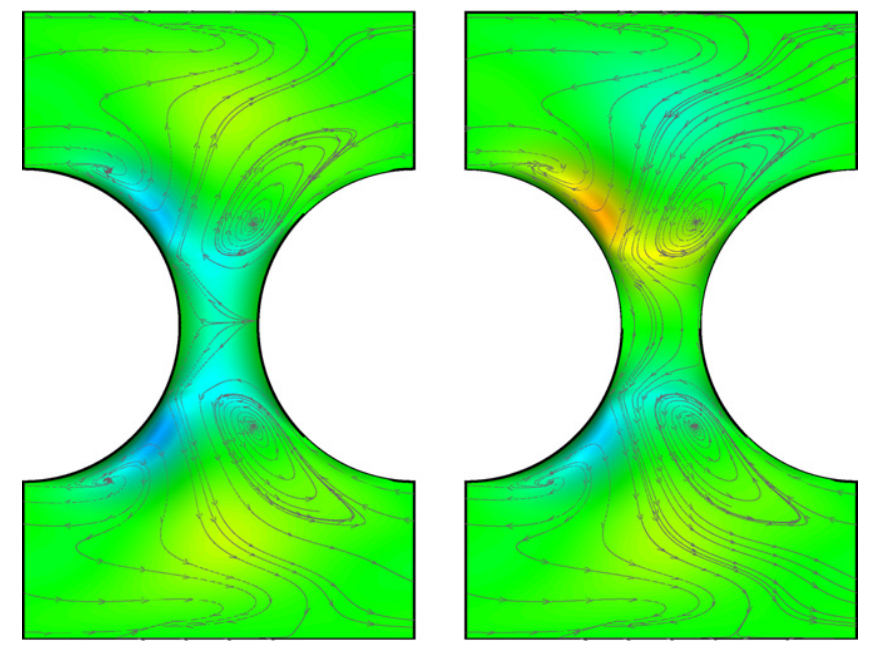

Fig. 6. The computed streamtraces with the $\hat{w}$-velocity component contours for the first two leading eigenfunctions on mesh M4 at a subcritical Weissenberg number of 1.45 with $L=2.5 R$ and $H=4.0 R$. The second eigenfunction (right) shows mass flow between the cylinders (Oldroyd-B fluid with $\beta=0.67$ ).
Table 3

Convergence of critical Weissenberg number and wavenumber with mesh refinement for $L=2.5 R$ and $H=4.0 R$ (Oldroyd-B fluid with $\beta=0.67$ )

\begin{tabular}{llllll}
\hline Mesh & $L=2.5 R$ & & & \multicolumn{2}{l}{$L=3.0 R$} \\
\cline { 2 - 3 } & $W e_{\text {crit }}$ & $k_{\text {crit }}$ & & $W e_{\text {crit }}$ & $k_{\text {crit }}$ \\
\hline M1 & 1.4667 & 3.0 & 1.5693 & 2.4 \\
M2 & 1.4812 & 3.0 & 1.5811 & 2.4 \\
M3 & 1.4884 & 3.0 & 1.5841 & 2.4 \\
M4 & 1.4913 & 3.0 & 1.5849 & 2.4 \\
\hline
\end{tabular}

The real three-dimensional flows can be constructed by means of a simple superposition of the first leading eigenvector on the base state:

$$
\begin{aligned}
& u=U(x, y)-\epsilon \hat{u}(x, y) \sin (k z), \\
& v=V(x, y)-\epsilon \hat{v}(x, y) \sin (k z), \\
& w=\epsilon \hat{w}(x, y) \cos (k z)
\end{aligned}
$$

where $\epsilon$ is a very small number. The reconstructed flow pattern at $W e=1.50$ is depicted in Fig. 7, indicating spiral-shaped vortices between the cylinders.

In order to determine the dependence of the onset of instabilities on the cylinder spacing, the cylinder spacing is increased to $L=3.0 R$. The computed $U$-velocity component, with streamtraces, and the extra stress component $T_{x x}$ are presented in Fig. 8 on mesh M4 at a Weissenberg number of 1.55 for $L=3.0 R$. The recirculation region between the cylinders shrinks and splits into two smaller circulation regions attached the fore and aft of the cylinder along the channel symmetry line. The computed eigenspectrum at this Weissenberg number predicts one symmetric and one antisymmetric leading eigenfunction as shown in Fig. 9. These are very similar to what is observed for $L=2.5 R$. However, the calculations indicate a critical Weissenberg number of 1.58 which is higher than the value at $L=2.5 R$. In addition, the critical wavenumber drops to a value of 2.4 . These values are in accord with the numerical results of Smith et al. [36].

The second set of numerical results corresponds to the flow of an Oldroyd-B fluid through a linear array of circular cylinders with wide cylinder spacing $(L=4.0 R$ and $6.0 R)$. For a cylinder spacing of $L=4.0 R$ two different meshes are employed: mesh M1 with 30,753 node points and 30,373 elements, and mesh M2 with 50,729 node points and 50,229 elements. The meshes are stretched along the channel symmetry line similar to the solid walls. Special attention has been paid to having element centers along the channel center line. In addition to mesh stretching, we made two more levels of mesh refinement along the channel

Table 4

\begin{tabular}{|c|c|c|c|c|c|c|c|c|}
\hline & \multicolumn{2}{|c|}{$L=2.5 R$} & \multicolumn{2}{|c|}{$L=3.0 R$} & \multicolumn{2}{|c|}{$L=4.0 R$} & \multicolumn{2}{|c|}{$L=6.0 R$} \\
\hline & $W e_{\text {crit }}$ & $k_{\text {crit }}$ & $W e_{\text {crit }}$ & $k_{\text {crit }}$ & $W e_{\text {crit }}$ & $k_{\text {crit }}$ & $W e_{\text {crit }}$ & $k_{\text {crit }}$ \\
\hline Liu & 1.53 & 3.1 & - & - & - & - & 1.36 & 4.0 \\
\hline Smith et al. & 1.55 & 3.1 & 1.64 & 2.5 & - & - & - & - \\
\hline Present (M4) & 1.49 & 3.0 & 1.58 & 2.4 & - & - & - & - \\
\hline
\end{tabular}

Comparison of computed critical Weissenberg number and critical wavenumber with several other results in the literature 


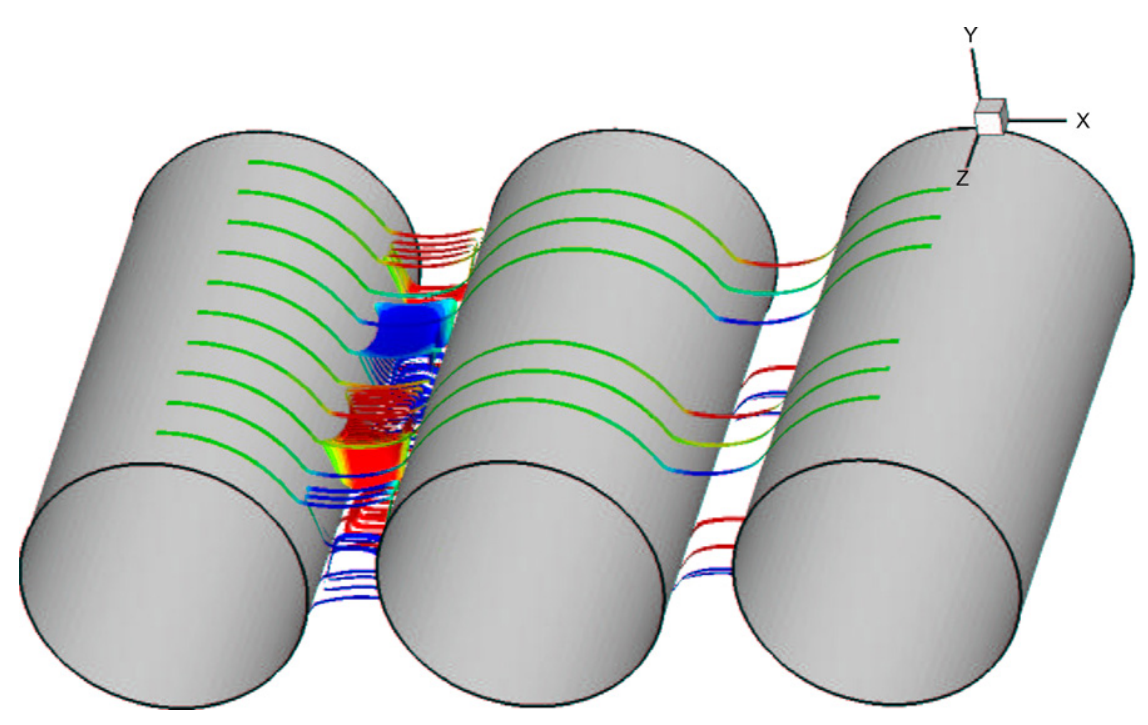

Fig. 7. Three-dimensional streamtraces reconstructed by means of superposition of the first leading eigenfunction and base flow at $W e=1.50$ for flow of an Oldroyd-B fluid $(\beta=0.67)$ past a confined circular cylinder in a rectangular channel with $L=2.5 R$ and $H=4.0 R$.
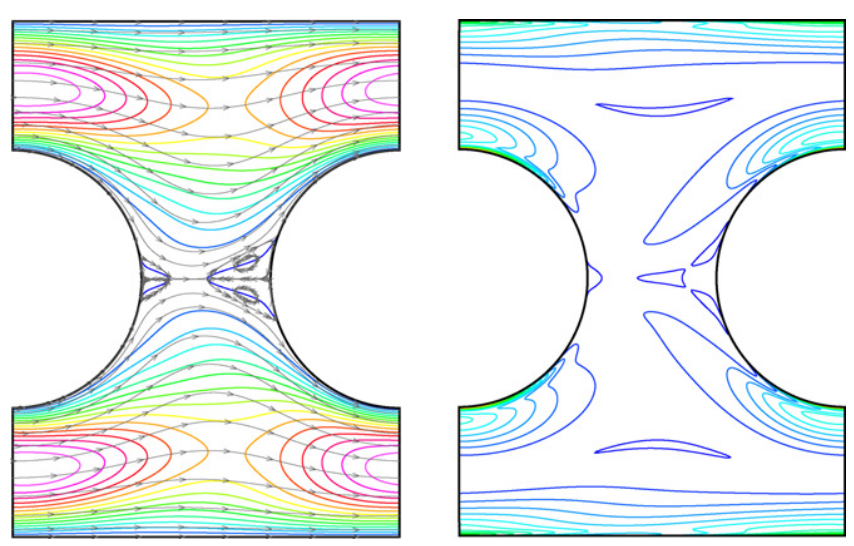

Fig. 8. Computed steady state $U$-velocity contours with streamlines (left) and $T_{x x}$ contour plots (right) on mesh M4 at a subcritical Weissenberg number of 1.55 for an Oldroyd-B fluid $(\beta=0.67)$ with $L=3.0 R$ and $H=4.0 R$. The contour levels shown are from 0 to 3 with an interval of 0.2 for the $U$-velocity and from 0 to 200 with an interval of 10 for $T_{x x}$.
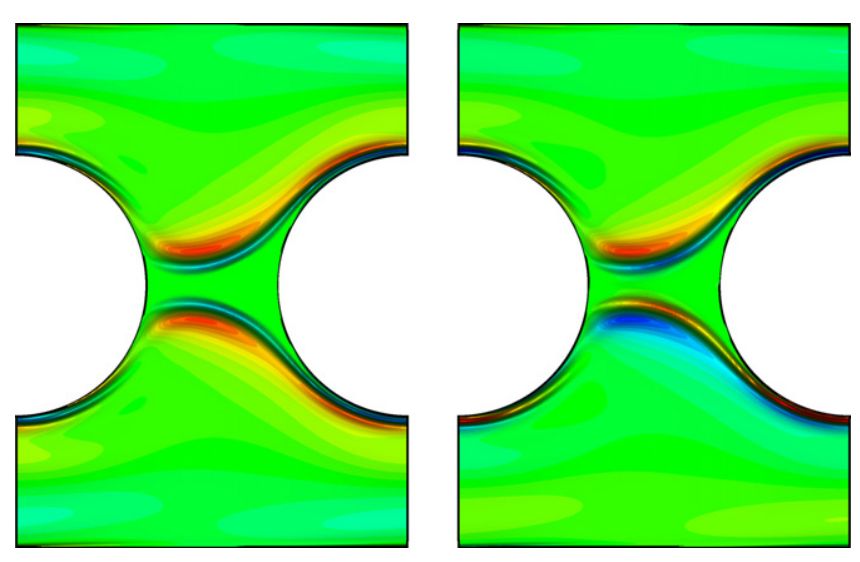

Fig. 9. The computed contours of $\hat{\tau}_{x x}$ for the first two leading eigenfunctions on mesh M4 at a subcritical Weissenberg number of 1.55 with $L=3.0 R$ and $H=4.0 R$. The first eigenfunction (left) is symmetric and the second one (right) is antisymmetric (Oldroyd-B fluid with $\beta=0.67$ ). symmetry line in order to resolve the wake adequately. Then two more additional mesh refinements were carried out just behind the first cylinder, where we first observed the high Weissenberg number problem (HWNP). The details of mesh M1 are shown in Fig. 10. The smallest mesh size on mesh M1 is reduced to $\Delta x_{\min }=0.00016 R$ and $\Delta y_{\min }=0.00025 R$. Although we made a significant reduction in mesh size, the results indicate only a small delay in the HWNP with mesh refinement. Therefore we were not able to compute the critical Weissenberg and wavenumbers exactly. However, we were able to show mesh independent results close to the Weissenberg numbers at which the HWNP occurs. The computed $U$-velocity component, with streamtraces, and the extra stress component $T_{x x}$ are presented in Fig. 11 on mesh M2 at a Weissenberg number of 1.45. At this cylinder spacing the circulation region between the cylinders completely disappears, and extremely large extensional stresses develop along the channel symmetry line. This is very similar to what is observed for a single cylinder in a channel. A further increase in Weissenberg number beyond 1.47 and 1.46 (on meshes M1 and M2, respectively) leads to divergence of the base flow solution. We could not afford the finer meshes M3 and M4 due to an excessive increase in the memory expansion parameter of the MUMPS library during LU decomposition. The change of the real leading eigenvalue as a function of wavenumber $k$ is given in Fig. 12 at Weissenberg numbers 1.40 and 1.45 . It may be seen that the growth rate becomes maximum around $k=40$ at a Weissenberg number of 1.45 . The calculation at $W e=1.40$ indicates a further increase in the critical wavenumber. However, we could not compute the leading eigenvalues at $W e=1.45$ on mesh M2 due to an excessive increase in the memory expansion parameter during the LU decomposition of the linear stability equations at sub-critical Weissenberg numbers. These wavenumbers are quite different from what is observed for close cylinder spacing. However, we observe a second extreme around $k=2.1$ which is close to that of the close cylinder spacing. It would appear that for close cylinder spacing the critical wavelength scales with 

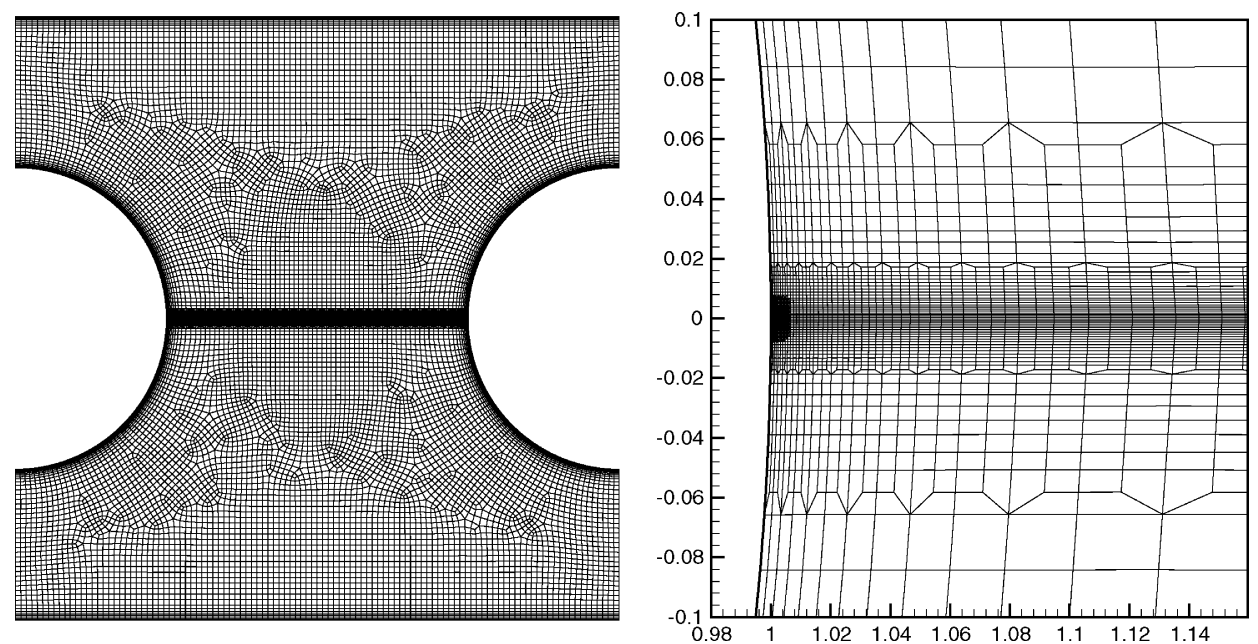

Fig. 10. The locally refined coarse mesh M1 for the flow past a confined periodic array of circular cylinders for $L=4.0 R$ and $H=4.0 R$ with 30,753 nodes and 30,373 elements. The details of mesh refinement are shown on the right just behind the first cylinder.

$L$, the cylinder spacing $(k L / 2 \pi$ is order 1 in Table 3$)$ whereas for the larger spacing, the wavelength is much smaller, on the same scale as the width of the highly stressed wake behind each cylinder.

The computed $\hat{w}$-velocity component of the leading eigenfunction at a Weissenberg number of 1.45 and $k=40$ is given in Fig. 13. The leading eigenfunction indicates very large spanwise velocity components confined to a very small region just behind the first cylinder, and this velocity component changes its direction over a relatively short distance. This large spanwise velocity component leads to the merging of streamtraces behind the circular cylinders, which is very similar to the experimental observations of McKinley et al. [24] and Shiang et al. [34] for a single cylinder. In addition to a very large $\hat{w}$-velocity component, a very large $\hat{u}$-velocity component is also observed along the channel symmetry line. The critical wavenumber at $W e=1.45$ indicates a wavelength $\lambda_{z}=0.16$ in the third dimension, which is very close to the width of the viscoelastic wake along the channel symmetry line and the width of the region of very large spanwise velocity shown in Fig. 13. It is interesting to
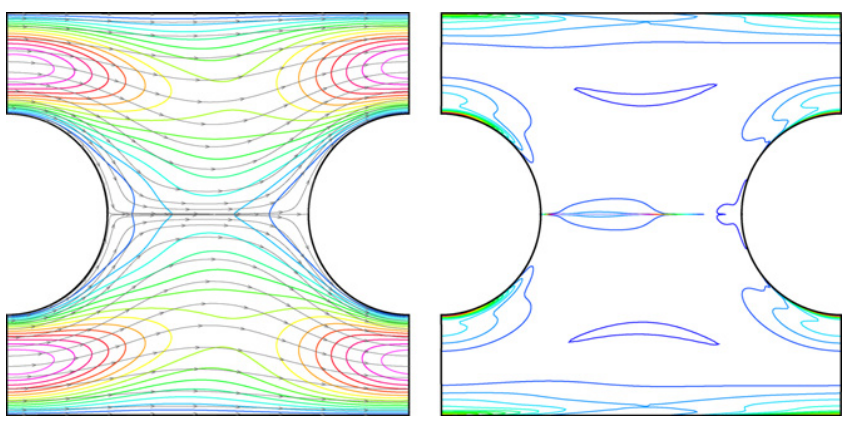

Fig. 11. Computed steady state $U$-velocity contours with streamlines (left) and $T_{x x}$ contour plots (right) on mesh M2 at a subcritical Weissenberg number of 1.45 for an Oldroyd-B fluid $(\beta=0.67)$ with $L=4.0 R$ and $H=4.0 R$. The contour levels shown are from 0 to 3 with an interval of 0.2 for the $U$-velocity and from 0 to 200 with an interval of 10 for $T_{x x}$. note that the large spanwise velocity component occurs where the streamline curvature changes sharply, probably indicating that these instabilities are related to the instability mechanism described by McKinley et al. [23] and Pakdel and McKinley [28] rather than being purely due to extremely large elongational stresses in the wake as suggested by Smith et al. [36]. Based on our calculations at $W e=1.40$ and 1.45 we expect the critical Weissenberg number to be around 1.5.

A further increase in cylinder spacing to $L=6.0 R$ also shows similar leading eigenfunctions at subcritical Weissenberg numbers. However, the extra stresses between the cylinders become extremely large at lower Weissenberg numbers. The calculations on meshes M1 and M2 did not converge beyond $W e=0.97$ and 0.90 , respectively. The calculations at higher Weissenberg numbers with M2 showed numerical oscillations across the wake, rather than oscillations behind the first cylinder, due to the extremely large extra stresses in the wake. We present the change

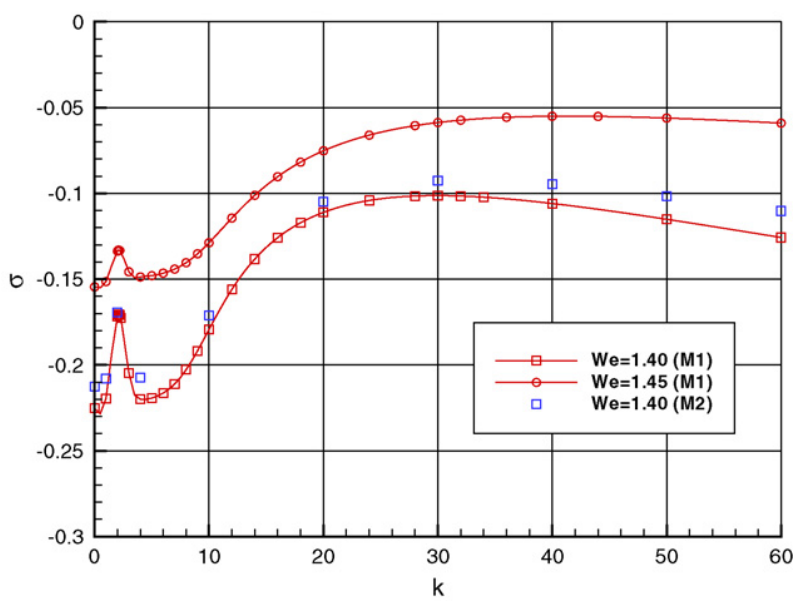

Fig. 12. The change of the leading real eigenvalue with the wave number $k$ on meshes M1 and M2 for the flow past a confined periodic array of circular cylinders with $L=4.0 R$ and $H=4.0 R$. 


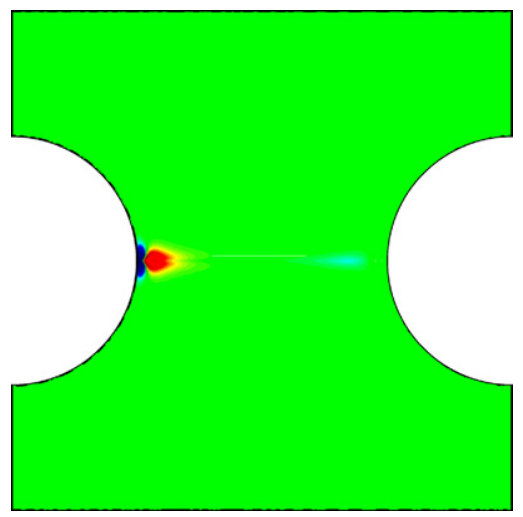

Fig. 13. The computed contours of $\hat{w}$-velocity for the first leading eigenfunction ( $k=40.0)$ on mesh M2 at a subcritical Weissenberg number of 1.45 with $L=$ $4.0 R$ and $H=4.0 R$.

of the leading real eigenvalue as a function of wavenumber $k$ at Weissenberg numbers 0.90 and 0.95 in Fig. 14. The calculations at $W e=0.95$ indicate maximum growth at $k=30$ which is lower than the value at $L=4.0 R$. The second extreme value is observed at $k=0.0$. The two-dimensional leading eigenfunction is given in Fig. 15. It is quite interesting that the leading two-dimensional eigenfunction predicts two small counter rotating vortices just behind the cylinder surface along the channel symmetry line, which is very similar to the small time-periodic separation bubble seen in the work of Oliveira and Miranda [26] for the FENE-CR model with $L^{2}=144$. Based on these calculations at $W e=0.90$ and 0.95 we estimate a critical Weissenberg number around 1.0. This would be a significant drop in the critical Weissenberg number with increase in cylinder spacing. However, the experimental results of Liu [21] predicted a critical Weissenberg number of 1.36 with a wavenumber of 4.0 for $L=6.0 R$; our lower value is due to the extremely large extra stresses in the wake predicted by the Oldroyd-B model. Further numerical work is needed in order to investigate the effect of finite extensibility properties of the viscoelastic fluid

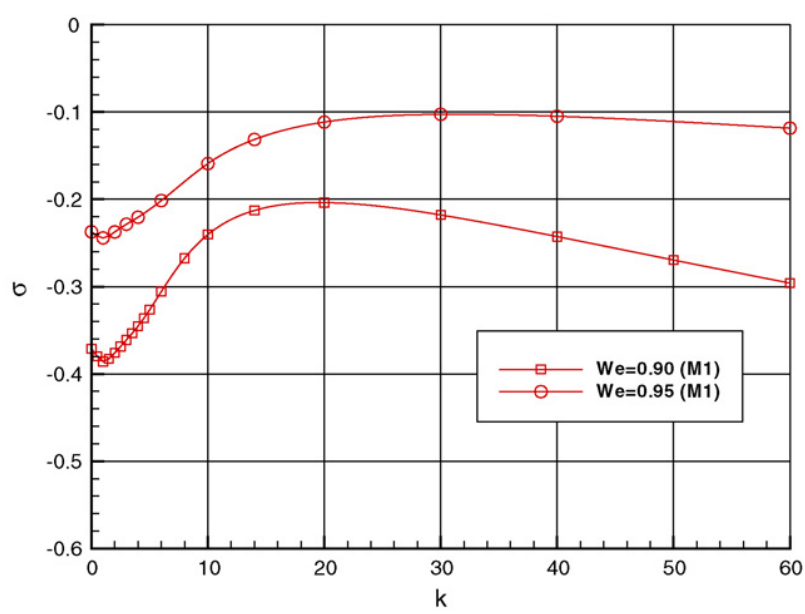

Fig. 14. The change of the leading real eigenvalue with the wavenumber $k$ on meshe M1 for the flow past a confined periodic array of circular cylinders with $L=6.0 R$ and $H=4.0 R$

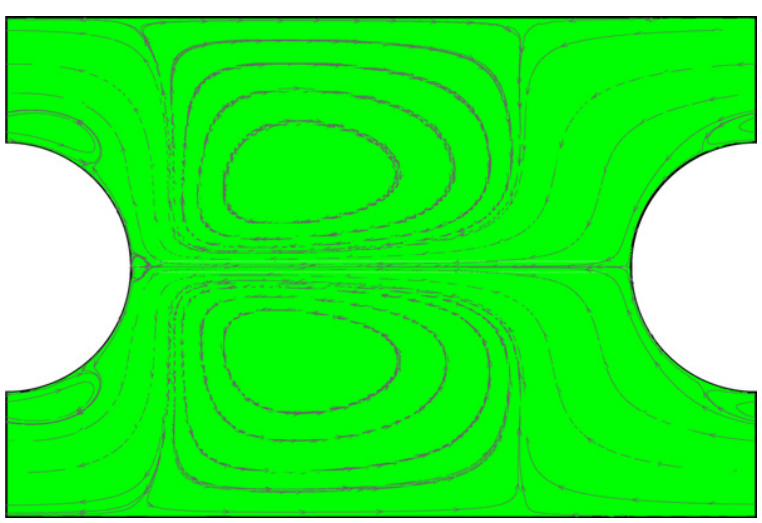

Fig. 15. The streamtraces of the two-dimensional $(k=0.0)$ leading eigenfunction on mesh M2 at a subcritical Weissenberg number of 0.90 with $L=6.0 R$ and $H=4.0 R$. The $\hat{w}$-velocity component is zero.

to the onset of three-dimensional instabilities, similar to the two-dimensional work of Sahin and Owens [31].

\subsection{Periodic array of half circular cylinders placed on channel walls}

Numerical simulations are carried out for the flow of an Oldroyd-B fluid past an array of circular half cylinders placed on the channel walls, for cylinder spacing $L=2.5 R$ and $6.0 R$. These calculations are relevant to a general class of contraction-expansion flows which are often encountered in polymer processing. The computed $U$-velocity component, with streamtraces, and the extra stress component $T_{x x}$ are presented in Fig. 16 for $L=2.5 R$ at a Weissenberg number of 2.70 on mesh M3 with 37,590 vertices and 37,129 elements. A separation region is observed between the cylinders, similar to the periodic array of circular cylinders. However, a significant pressure drop is observed between the inlet and outlet
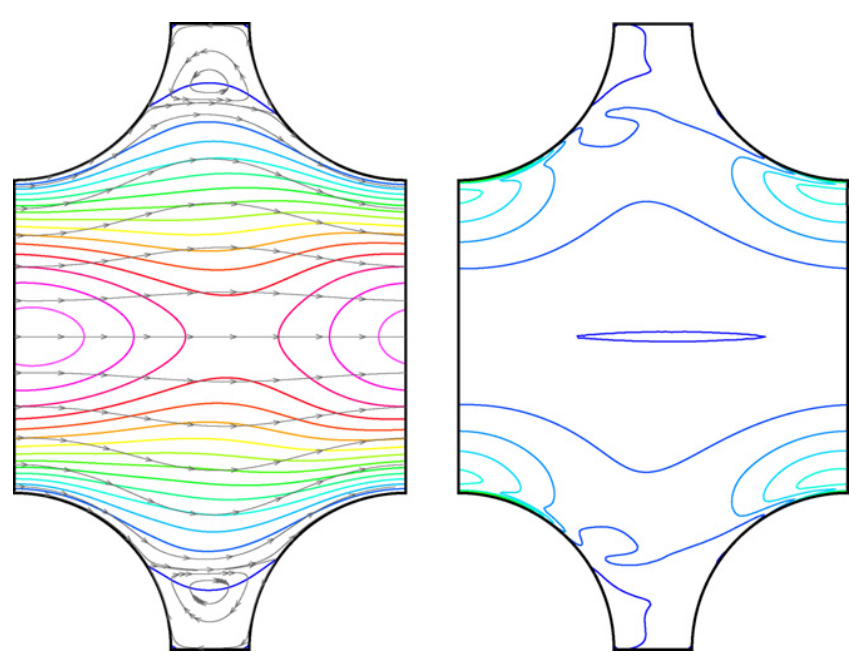

Fig. 16. Computed steady state $U$-velocity contours with streamlines (left) and $T_{x x}$ contour plots (right) on mesh M3 at a subcritical Weissenberg number of 2.70 for an Oldroyd-B fluid $(\beta=0.67)$ with $L=2.5 R$ and $H=4.0 R$. The contour levels shown are from 0 to 3 with an interval of 0.2 for the $U$-velocity and from 0 to 200 with an interval of 10 for $T_{x x}$. 
as well as an extreme value of the extra stresses. The linear stability calculations predict a single real leading eigenvalue which is well separated from the rest of the eigenspectrum. The perturbations with $k_{\text {crit }}=2.0$ are the fastest growing and the growth rate becomes positive beyond $W e_{\text {crit }}=2.73$. The critical Weissenberg number is relatively larger than the values observed for periodic array of cylinders. The computed contours of $\hat{\tau}_{x x}$ and the $\hat{w}$-velocity component, with streamtraces, are shown for the first leading real eigenfunction in Fig. 17. The leading eigenfunction is antisymmetric along the channel center line. The $\hat{w}$-velocity component of the leading eigenfunction changes its direction immediately behind the first cylinder and at the front of second cylinder. The constructed three-dimensional flow field on the basis of two-dimensional steady base flow with the leading first eigenfunction is depicted in Fig. 18 and indicates spiral-shaped vortices between the cylinders. These are very similar to what is observed for the linear array of confined cylinders. However, the streamtraces are shifted in the spanwise direction for the upper and the lower cylinders.

To show the effect of cylinder spacing, the distance between the cylinders is increased to $L=6.0 R$ and similar calculations are carried out. For this cylinder spacing the separation vortices between the cylinders are completely eliminated. Only very small corner eddies are observed at the junction between the cylinder and the wall. The linear stability calculations predict a critical Weissenberg number $W_{\text {crit }}=4.56$ with a wavenumber $k_{\text {crit }}=1.4$ on mesh M3 with 103,194 vertices and 102,513 elements, indicating a significant increase in the critical Weissenberg number, at the same time as a decrease in wavenumber $k$. The leading antisymmetric eigenfunction shown in Fig. 19 at $W e=4.55$ is very similar to that of $L=2.5 R$. However,
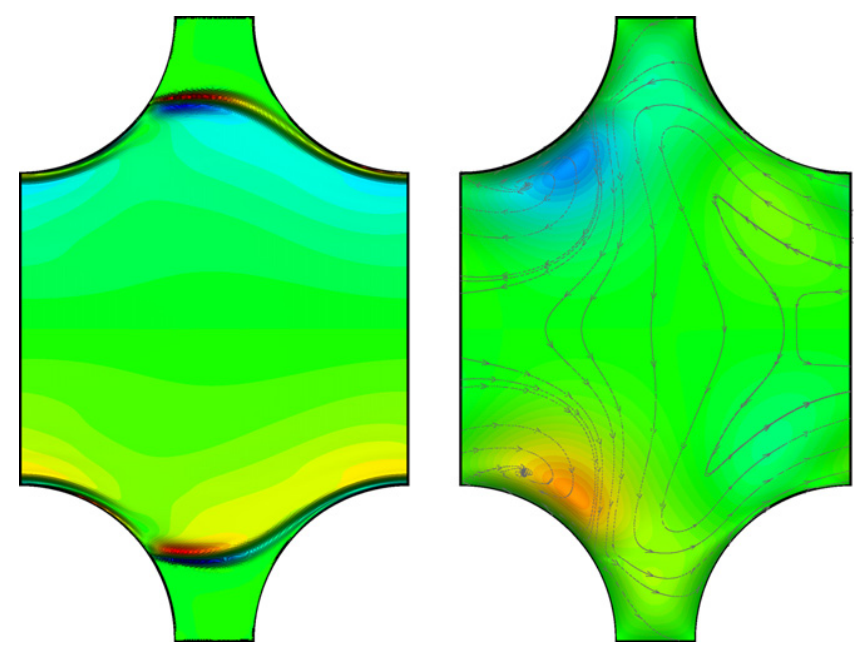

Fig. 17. The computed contours of $\hat{\tau}_{x x}$ (left) and $\hat{w}$-velocity contours with streamtraces (right) for the first leading eigenfunction $\left(k_{\text {crit }}=2.0\right)$ on mesh M3 at a subcritical Weissenberg number of 2.70 with $L=2.5 R$ and $H=4.0 R$ (Oldroyd-B fluid with $\beta=0.67$ ).

there are more evident spiral-type vortices in front of the second cylinder. The $\hat{w}$-velocity components on the upper and lower cylinders are in opposite directions, giving rise to a rotation of the viscoelastic fluid about the channel symmetry line. This may explain spiral-type instabilities which are observed during some extrusion processing. In addition to the current calculations, we have placed half cylinders asymmetrically on the channel walls; this did not alter the numerical results significantly. However, a decrease in channel height $H$ can significantly reduce the critical Weissenberg number while increasing the wavenumber $k$.

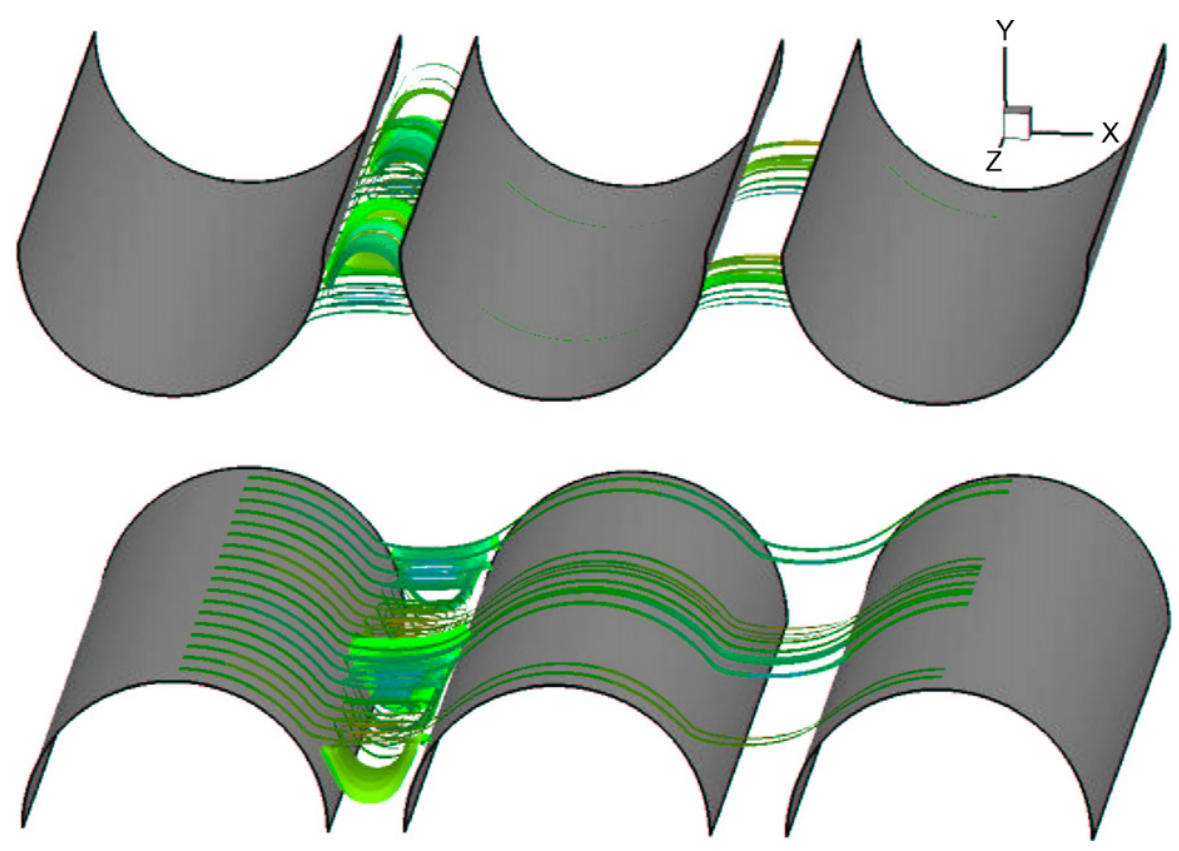

Fig. 18. Three-dimensional streamtraces reconstructed by means of superposition of the first leading eigenfunction and base flow at $W e=2.80$ for flow of an Oldroyd-B fluid $(\beta=0.67)$ past a confined circular cylinder in a rectangular channel with $L=2.5 R$ and $H=4.0 R$. 

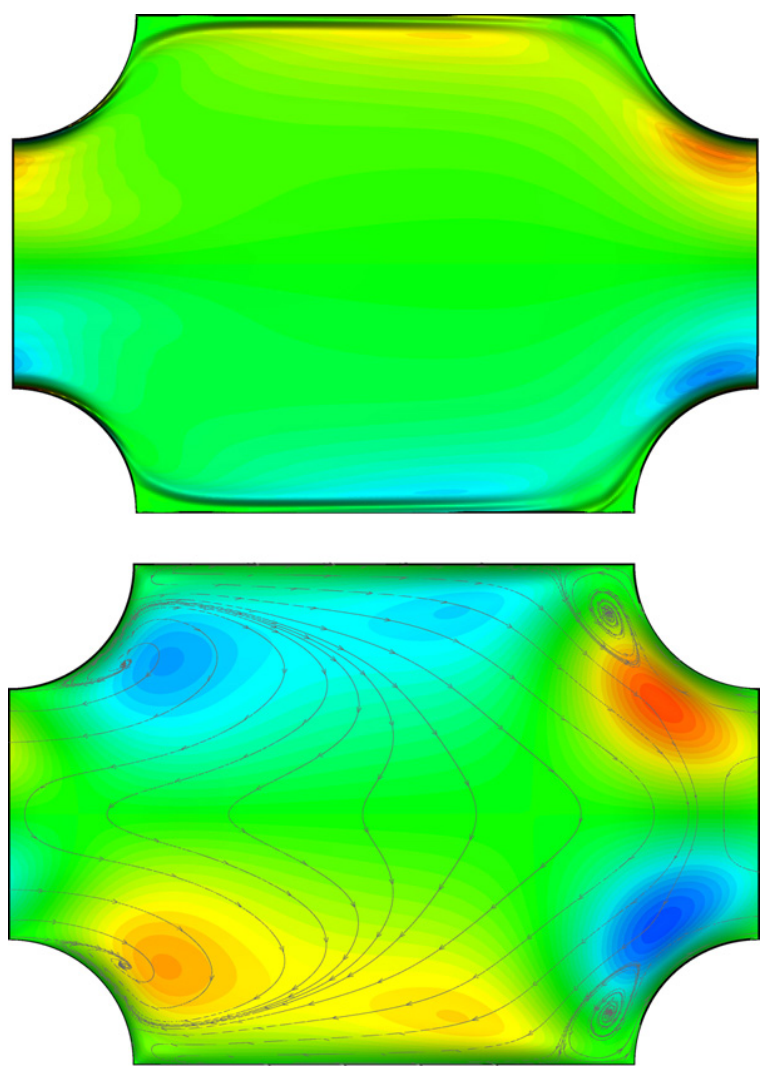

Fig. 19. The computed contours of $\hat{\tau}_{x x}$ (upper) and $\hat{w}$-velocity component with streamtraces (lower) for the first leading eigenfunctions $\left(k_{\text {crit }}=1.4\right)$ on mesh M3 at a subcritical Weissenberg number of 4.55 with $L=6.0 R$ and $H=4.0 R$. (Oldroyd-B fluid with $\beta=0.67$ ).

\section{Conclusions}

An efficient numerical scheme based on semi-staggered finite volume is presented for the linear stability analysis of twodimensional steady Oldroyd-B fluid flows to small amplitude, three-dimensional perturbations. The use of the highly efficient MUMPS library combined with the shift invert Arnoldi method allows us to solve very large generalized eigenvalue problems (order of a million) on parallel machines. The proposed method is applied to the linear stability analysis of viscoelastic flow around a periodic linear array of confined circular cylinders and a periodic array of circular half cylinders placed on channel walls. Two different leading eigenfunctions are identified for close and wide cylinder spacing for the periodic array of cylinders. The wavelength of the instability scales with the cylinder spacing for close cylinders, and with the wake width for wellseparated cylinders. The results indicate good agreement with the numerical and experimental results available in the literature.

\section{Acknowledgments}

This work was supported by EPSRC Grant GR/T11807/01. The authors acknowledge the use of the SGI Altix 3700 machine at UCL Research Computing.

\section{References}

[1] P.R. Amestoy, I.S. Duff, J.-Y. L'Excellent, Multifrontal parallel distributed symmetric and unsymmetric solvers, Comput. Methods Appl. Mech. Eng. 184 (2000) 501-520.

[2] P.R. Amestoy, I.S. Duff, J. Koster, J.-Y. L'Excellent, A fully asynchronous multifrontal solver using distributed dynamic scheduling, SIAM J. Matrix Anal. Appl. 23 (2001) 15-41.

[3] W.K. Anderson, D.L. Bonhaus, An implicit upwind algorithm for computing turbulent flows on unstructured grids, Comput. Fluids 23 (1994) $1-21$.

[4] W.E. Arnoldi, The principle of minimized iterations in the solution of the matrix eigenvalue problem, Quart. Appl. Math. 9 (1951) 17-29.

[5] K. Arora, R. Sureshkumar, B. Khomami, Experimental investigation of purely elastic instabilities in periodic flows, J. Non-Newton. Fluid Mech. 108 (2002) 209-226.

[6] T.J. Barth, A 3-D upwind Euler solver for unstructured meshes, AIAA Paper 91-1548-CP, 1991.

[7] T.D. Blacker, S. Benzley, S. Jankovich, R. Kerr, J. Kraftcheck, R. Kerr, P. Knupp, R. Leland, D. Melander, R. Meyers, S. Mitchell, J. Shepard, T. Tautges, D. White, CUBIT Mesh Generation Environment Users' Manual, vol. 1, Sandia National Laboratories, Albuquerque, NM, 1999.

[8] T.D. Blacker, M.B. Stephenson, Paving: A New Approach to Automated Quadrilateral Mesh Generation. SAND90-0249, Sandia National Laboratories, Albuquerque, NM, 1990.

[9] J.A. Byars, Experimental characterization of viscoelastic flow instabilities, $\mathrm{PhD}$ dissertation, Massachusetts Institute of Technology, Cambridge, MA, USA, 1996.

[11] C. Chauviere, R.G. Owens, How accurate is your solution? Error indicators for viscoelastic flow calculations, J. Non-Newton. Fluid Mech. 95 (2000) $1-33$.

[12] C. Chmielewski, K. Jayaraman, Elastic instability in crossflow of polymer solutions through periodic arrays of cylinders, J. Non-Newton. Fluid Mech. 48 (1993) 285-301.

[13] Y. Ding, M. Kawahara, Linear stability of incompressible flow using a mixed finite element method, J. Comput. Phys. 139 (1998) 243273.

[14] V.J. Ervin, L.N. Ntasin, A posteriori error estimation and adaptive computation of viscoelastic fluid flow, J. Non-Newton. Fluid Mech. 48 (1993) 285-301.

[15] A. Fortin, F. Bertrand, M. Fortin, E. Chamberland, P.E. BoulangerNadeau, A.El. Maliki, N. Najeh, An adaptive remeshing strategy for shear-thinning fluid flow simulations, Comp. Chem. Eng. 28 (2004) 2363-2375.

[16] G. Georgiou, S. Momami, M.J. Crochet, K. Walters, Newtonian and nonNewtonian flow in a channel obstructed by an antisymmetric array of cylinders, J. Non-Newton. Fluid Mech. 40 (1991) 231-260.

[17] M.A. Hulsen, R. Fattal, R. Kupferman, Flow of viscoelastic fluids past a cylinder at high Weissenberg number: stabilized simulations using matrix logarithms. J. Non-Newton. Fluid Mech. 127 (2005) 2729.

[18] J.M. Kim, C. Kim, K.H. Ahn, S.J. Lee, An efficient iterative solver and high-resolution computations of the Oldroyd-B fluid flow past a confined cylinder, J. Non-Newtonian Fluid Mech. 123 (2004) 161-173.

[19] B. Khomami, L.D. Moreno, Stability of viscoelastic flow around periodic arrays of cylinders, Rheol. Acta 36 (1997) 367-383.

[20] R.G. Larson, Instabilities in viscoelastic flows, Rheol. Acta 31 (1992) 213-263.

[21] A.W. Liu, Viscoelastic flow of polymer solutions around arrays of cylinders: comparison of experiment and theory, $\mathrm{PhD}$ dissertation, Massachusetts Institute of Technology, Cambridge, MA, USA, 1997.

[22] A.W. Liu, D.E. Bornside, R.C. Armstrong, R.A. Brown, Viscoelastic flow of polymer solutions around periodic linear array of cylinders: comparisons of predictions for microstructure and flow fields, J. Non-Newton. Fluid Mech. 77 (1998) 153-190.

[23] G.H. McKinley, P. Pakdel, A. Öztekin, Rheological and geometric scaling of purely elastic flow instabilities, J. Non-Newton. Fluid Mech. 67 (1996) 19-47. 
[24] G.H. McKinley, R.C. Armstrong, R.A. Brown, The wake instability in viscoelastic flow past confined circular cylinders, Phil. Trans. R. Soc. Lond. A 344 (1993) 265-304.

[25] I. Mutlu, P. Townsend, M.F. Webster, Adaptive solutions for viscoelastic flows, Comm. Numer. Methods Eng. 12 (1996) 643-655.

[26] P.J. Oliveira, A.I.P. Miranda, A numerical study of steady and unsteady viscoelastic flow past bounded cylinders, J. Non-Newton. Fluid Mech. 127 (2005) 51-66

[27] S.J. Owen (Ed.), Cubit 9.0 Users Manual, Sandia Report SAND94-1100 Rev. 4/2002, Sandia National Laboratories, June 2004.

[28] P. Pakdel, G.H. McKinley, Elastic instabilities and curved streamlines, Phys. Rev. Lett. 77 (1996) 2459-2462.

[29] Y. Saad, Variations on Arnoldi's method for computing eigenelements of large non-symmetric matrices, Linear Algebra Appl. 34 (1980) 269-295.

[30] M. Sahin, A preconditioned semi-staggered dilation-free finite volume method for the incompressible Navier-Stokes equations on all-hexahedral elements, Int. J. Numer. Meth. Fluids 49 (2005) 959-974.

[31] M. Sahin, R.G. Owens, On the effects of viscoelasticity on two-dimensional vortex dynamics in the cylinder wake, J. Non-Newton. Fluid Mech. 123 (2004) 121-139.

[32] M. Sahin, H.J. Wilson, A semi-staggered dilation-free finite volume method for the numerical solution of viscoelastic fluid flows on all-hexahedral elements, J. Non-Newton. Fluid Mech. 147 (2007) 79-91.
[33] E.S.G. Shaqfeh, Purely elastic instabilities in viscometric flows, Annu. Rev. Fluid Mech. 28 (1996) 129-229.

[34] A.H. Shiang, A. Oztekin, J.-C. Lin, D. Rockwell, Hydroelastic instabilities in viscoelastic flow past a cylinder confined in a channel, Exp. Fluids 28 (2000) 128-142.

[35] M.D. Smith, R.C. Armstrong, R.A. Brown, R. Sureshkumar, Finite element analysis of stability of two-dimensional viscoelastic flows to three-dimensional perturbations, J. Non-Newton. Fluid Mech. 93 (2000) 203-244.

[36] M.D. Smith, Y.L. Joo, R.C. Armstrong, R.A. Brown, Linear stability analysis of flow of an Oldroyd-B fluid through a linear array of cylinders, $\mathrm{J}$. Non-Newton. Fluid Mech. 109 (2003) 13-50.

[37] A. Souvaliotis, A.N. Beris, Spectral collacation/domain decomposition method for viscoelastic flow simulations in model porous geometries, Comput. Methods Appl. Mech. Eng. 129 (1996) 9-28.

[38] R. Sureshkumar, M.D. Smith, R.C. Armstrong, R.A. Brown, Linear stability and dynamics of viscoelastic flows using time-dependent numerical simulations, J. Non-Newton. Fluid Mech. 82 (1999) 57-104.

[39] K.K. Talwar, B. Khomami, Flow of viscoelastic fluids past periodic square arrays of cylinders: inertial and shear thinning viscosity and elasticity effects, J. Non-Newton. Fluid Mech. 57 (1995) 177-202. 